\title{
ARTICLE
}

\section{Virus-induced accumulation of intracellular bile acids activates the TGR5- $\beta$-arrestin-SRC axis to enable innate antiviral immunity}

\author{
Ming-Ming $\mathrm{Hu}^{1,2,3}$, Wen-Rui $\mathrm{He}^{2}$, Peng Gao ${ }^{2}$, Qing Yang ${ }^{2}$, Ke He${ }^{4}$, Li-Bo Cao ${ }^{5}$, Shu $\mathrm{Li}^{1,2}$, Yu-Qi Feng (D) ${ }^{4}$ and Hong-Bing Shu (D) ${ }^{1,2}$
}

The mechanisms on metabolic regulation of immune responses are still elusive. We show here that viral infection induces immediate-early NF-кB activation independent of viral nucleic acid-triggered signaling, which triggers a rapid transcriptional induction of bile acid (BA) transporter and rate-limiting biosynthesis enzymes as well as accumulation of intracellular BAs in divergent cell types. The accumulated intracellular BAs activate SRC kinase via the TGR5-GRK- $\beta$-arrestin axis, which mediates tyrosine phosphorylation of multiple antiviral signaling components including RIG-I, VISA/MAVS, MITA/STING, TBK1 and IRF3. The tyrosine phosphorylation of these components by SRC conditions for efficient innate antiviral immune response. Consistently, TGR5 deficiency impairs innate antiviral immunity, whereas BAs exhibit potent antiviral activity in wild-type but not TGR5-deficient cells and mice. Our findings reveal an intrinsic and universal role of intracellular BA metabolism in innate antiviral immunity.

Cell Research (2019) 29:193-205; https://doi.org/10.1038/s41422-018-0136-1

\section{INTRODUCTION}

In the past decade, remarkable advances have been made in elucidating the molecular mechanisms of host antiviral defense. Upon viral infection, viral nucleic acids are sensed by cytosolic pattern recognition receptors (PRRs), including the helicase proteins RIG-I/MDA5 for viral RNA and the cyclic GMP-AMP synthase cGAS for viral and other cytosolic DNA. ${ }^{1-3}$ Upon sensing of viral RNA, RIG-I/MDA5 are recruited to the mitochondrialassociated adaptor protein called VISA (also named MAVS, IPS-1 and Cardif) ${ }^{4-7}$ which in turn associates with TRAFs, and the kinases TBK1 and IKK, leading to activation of the transcription factors NF-KB and IRF3 as well as induction of downstream antiviral genes. ${ }^{2}$ On the other hand, sensing of cytosolic DNA by cGAS causes the synthesis of cyclic GMP-AMP (cGAMP) from GTP and $A T P,{ }^{8,9}$ which acts as a second messenger to bind to the ER membrane-associated adaptor protein called MITA (also named STING). ${ }^{10,11}$ MITA is then translocated from the ER via Golgi apparatus to perinuclear punctate structures. In this process, MITA recruits TBK1 and IRF3, leading to phosphorylation of IRF3 and induction of downstream antiviral genes. ${ }^{12}$ Although various reports have suggested that innate antiviral responses are regulated by post-translational modifications, such as phosphorylation, polyubiquitination and sumoylation, 3,13 how they are efficiently initiated and precisely regulated upon viral infection is still not fully understood.

In recent years, accumulated evidence points to inter-regulation of cellular metabolism and immune response. ${ }^{14,15}$ Bile acids (BAs) are a diverse class of cholesterol-derived, amphipathic molecules that function both as detergents to facilitate digestion and absorption of dietary lipids, and as hormones endowed with systemic endocrine functions. ${ }^{16,17}$ Due to the extremely low levels of several rate-limiting enzymes (such as CYP7A1, CYP7B1, and CYP27A1) involved in BA biosynthesis in many extrahepatic organs, the biosynthesis of BAs is believed to be exclusively limited to hepatic tissues. ${ }^{16}$ By binding to the Takeda G Protein Coupled Receptor-5 (TGR5, also known as GPBAR1 or GPR131) or the nuclear Farnesoid X Receptor (FXR), BAs have been reported to regulate various cellular events, such as metabolic processes, NLRP3 inflammasome activation, phagocytosis of hepatic macrophages, and viral hepatitis. ${ }^{18-24}$ However, all these functions are executed by BAs biosynthesized and secreted from the liver or absorbed from the intestine. Whether BA biosynthesis occurs in extra-hepatic cells and the underlying significance have rarely been explored. Furthermore, whether intracellular BA metabolism is intrinsically involved in antiviral immune response especially in extra-hepatic cells is still elusive.

In this study, we demonstrate that viral infection induces rapid expression of BA transporter and rate-limiting biosynthesis enzymes in an immediate-early NF-KB activation-dependent process, leading to intracellular accumulation of BAs in hepatic and non-hepatic cells. The accumulated BAs activate the TGR5GRK- $\beta$-arrestin-SRC axis, which causes tyrosine phosphorylation of key components in the RIG-I/MDA5- and cGAS-mediated pathways. The phosphorylation is required for the activation of these components and onset of efficient innate antiviral immune response. Consistently, TGR5 deficiency impairs innate antiviral immunity, whereas BAs induce expression of antiviral genes and exhibit potent antiviral activity in cells and mice. Our results suggest that virus-induced intracellular accumulation of BAs and subsequent activation of the TGR5-GRK- $\beta$-arrestin-SRC axis

\footnotetext{
${ }^{1}$ Department of Infectious Diseases, Zhongnan Hospital of Wuhan University, Wuhan 430071, China; ${ }^{2}$ Medical Research Institute, School of Medicine, Wuhan University, Wuhan 430071, China; ${ }^{3}$ State Key Laboratory of Virology, Wuhan University, Wuhan 430072, China; ${ }^{4}$ College of Chemistry and Molecular Sciences, Wuhan University, 430072 Wuhan, China and ${ }^{5}$ College of Life Sciences, Wuhan University, Wuhan 430072, China

Correspondence: Ming-Ming Hu (mmhu@whu.edu.cn) or Hong-Bing Shu (shuh@whu.edu.cn)
}

Received: 25 October 2018 Accepted: 20 December 2018

Published online: 16 January 2019 
194

promote innate antiviral immunity, and therefore reveal an intrinsic and universal role of intracellular BA metabolism in antiviral innate immunity.

\section{RESULTS}

Viral infection induces BA transporter and rate-limiting biosynthesis enzymes via immediate-early NF-KB activation To investigate the potential relationship between $B A$ metabolism and innate antiviral immunity, we examined the expression of genes involved in BA biosynthesis and uptake in human monocytic THP1 cells following viral infection. Both HSV-1 (DNA virus) and Sendai virus (SeV, RNA virus) specifically induced transcription of several critical rate-limiting enzymes involved in BA biosynthesis including CYP7A1, CYP7B1 and CYP27A1, as well as the plasma membrane-located BA transporter SLCO1B2 (Fig. 1a and Supplementary information, Fig. S1a). In these experiments, HSV-1 and SeV also induced transcription of the classic antiviral gene IFNB1, but did not induce transcription of the examined nonrate-limiting enzymes such as HSD3B7, SLC27A5, and AKR1D1 among others, or other transporters including SLC51A and SLC51B, which mainly form a heterodimer that acts as the intestinal basolateral transporter responsible for BA export from enterocytes into portal blood (Fig. 1a). In addition, the transcripts of several liver- and enterohepatic tissue-restricted genes including CYP8B1, SLC27A5, NTCP and ASTB were barely detected in THP1 cells before and after viral infection (Fig. 1a). Induction of these BA biosynthesis enzymes and transporter can also be triggered by other viruses, such as encephalomyocarditis virus (EMCV), vesicular stomatitis virus (VSV), newcastle disease virus (NDV), human cytomegalovirus (HCMV) and vaccinia virus (VV) (Supplementary information, Fig. S1b).

Viral infection activates the kinases $I K K a / \beta$ and TBK1, which activate NF-KB and IRF3 respectively, leading to induction of type I interferons (IFNs) and proinflammatory cytokines. Type I IFNs further induces downstream antiviral genes via JAK-STAT pathway. To investigate how viral infection induces BA transporter and ratelimiting biosynthesis enzymes, we examined the effects of the IKKa/ $\beta$ inhibitor BMS-345541, the TBK1 inhibitor MRT67307, and the JAKs inhibitor SAR-20347 on virus-induced transcription of BA transporter and rate-limiting biosynthesis enzyme genes. We found that BMS-345541 but not MRT67307 or SAR-20347 abolished HSV-1- and SeV-induced transcription of CYP7A1, CYP7B1, CYP27A1 and SLCO1B2 genes in THP1 cells (Fig. 1b). In the same experiments, all the three inhibitors impaired virusinduced transcription of IFNB1 gene (Fig. 1b). These results suggest that virus-triggered NF-KB activation is essential for induction of the $\mathrm{BA}$ transporter and rate-limiting biosynthesis enzyme genes. Consistently, CRISPR/Cas9-mediated knockout of the NF-KB transactivator p65 but not IRF3 dramatically inhibited SeV- and HSV-1-induced transcription of the CYP7A1, CYP7B1, CYP27A1 and SLCO1B2 genes as well as the well-known NF-KBtarget gene IKBA (Fig. 1C). CHIP assays indicated that viral infection induced the binding of p65 to the promoters of CYP7A1, CYP7B1, CYP27A1, SLCO1B2 and IKBA genes but not to intergenic regions of chromatins (Fig. 1d). These results suggest that viral infection induces expression of BA rate-limiting biosynthesis and transporter genes in an NF-KB-dependent process.

Viral infection can activate NF-KB by several distinct mechanisms, such as virus-cell membrane fusion, viral nucleic acidtriggered signaling, and expression of certain viral proteins. ${ }^{25}$ Our experiments indicated that deficiency of VISA/MAVS or MITA/ STING, which are essential adaptors in viral RNA- and DNAtriggered NF-KB and IRF3 activation pathways respectively, impaired SeV- and HSV-1-induced transcription of IFNB1 gene respectively, but had no marked effects on virus-induced transcription of CYP7A1, CYP7B1, CYP27A1, and SLCO1B2 genes (Fig. 1e). Interestingly, biochemical analysis showed that while
VISA or MITA deficiency abolished SeV- and HSV-1-induced phosphorylation of IRF3 respectively, their deficiencies only impaired the phosphorylation of $1 \mathrm{KKa} / \beta$ and $\mathrm{p} 65$, which are hallmarks of NF-KB activation, at the late phase (4-10 h) but not the immediate-early phase $(2 \mathrm{~h})$ after viral infection (Fig. 1f). Consistently, UV treatment of viruses, which impairs viral replication but not entry into the cell, impaired HSV-1- or SeVinduced transcription of IFNB1 gene but did not affect virusinduced transcription of the BA rate-limiting biosynthesis enzyme and transporter genes (Supplementary information, Fig. S1c). In addition, UV-treated viruses induced normal phosphorylation of $\mathrm{IKKa} / \beta$ and $\mathrm{p} 65$ at the immediate-early phase $(2 \mathrm{~h})$ of viral infection but failed to induce their phosphorylation at the late phase (4-10 h) (Supplementary information, Fig. S1d). In the same experiments, phosphorylation of IRF3 was induced at $4 \mathrm{~h}$ post $\mathrm{SeV}$ or HSV-1 infection in wild-type cells, which was abolished in VISAor MITA-deficient cells respectively, or by UV treatment of the viruses (Fig. If and Supplementary information, Fig. S1d). These results suggest that the immediate-early first-wave and VISA- or MITA-independent activation of NF-KB following viral infection drives the transcription of $\mathrm{BA}$ rate-limiting biosynthesis enzyme and transporter genes, and IRF3 activation occurs after the firstwave of VISA- and MITA-independent NF-KB activation.

Viral infection induces accumulation of intracellular BAs

Since viral infection induces expression of BA transporter and ratelimiting biosynthesis enzymes, we determined whether viral infection leads to accumulation of intracellular BAs. We found that SeV and HSV-1 infection for $3 \mathrm{~h}$ caused dramatic increase of intracellular BA levels from 7.4 $\mu \mathrm{M}$ to $63.1 \mu \mathrm{M}$ and $91.6 \mu \mathrm{M}$ respectively in human monocytic THP1 cells that are cultured in FBS-containing complete medium (CM) (Fig. 2a). Viral infectioninduced accumulation of intracellular $B A$ is not limited to THP1 cells, because similar results were also obtained in epithelial HEK293 cells as well as hepatic WRL68 cells (Supplementary information, Fig. S2a and b). Interestingly, the increase of BA levels after viral infection was $\sim 40 \%$ lower in cells cultured in FBS-free basic medium (BM) in comparison to CM (Fig. 2a and Supplementary information, Fig. S2a and b). Since CM contains BAs but BM does not, these results indicate that both de novo biosynthesis and extracellular to intracellular transportation contribute to virusinduced accumulation of intracellular BAs. Our results also showed that the BA levels were rapidly increased at the early phase (3-6 h) and then decreased at the late phase (12 h) after SeV and HSV-1 infection (Fig. 2a and Supplementary information, Fig. S2a and b), which is consistent with a critical role of BAs in conditioning innate antiviral response (see below). The only exception was that BA levels were continually increased by $12 \mathrm{~h}$ after SeV infection in hepatic WRL68 cells cultured in CM (Supplementary information, Fig. S2a). It is possible that hepatic cells respond longer for RNA virus-induced uptake of extracellular BAs. Furthermore, we found that viral infection induced increase of intracellular BA levels in mouse BMDMs and primary hepatocytes, while the extracellular BA levels in the medium were slightly decreased (Supplementary information, Fig. S2c).

Since BAs contain various primary and secondary molecules, we further determined which types of BAs were accumulated after viral infection by mass spectrometry (MS). We found that only the primary CDCA but not the secondary lithocholic acid (LCA) or DCA was dramatically increased in THP1 cells cultured in BM after SeV or HSV-1 infection (Fig. 2b), suggesting that CDCA but not LCA or DCA is biosynthesized after viral infection. However, all the three BAs were increased in THP1 cells cultured in CM after SeV or HSV-1 infection (Fig. 2b), suggesting that viral infection induces uptake from the medium of the secondary LCA and DCA. Similar results were also obtained in other cells including primary mouse hepatocytes and HEK293 (Supplementary information, Fig. S2d). In these experiments, we noticed that viral infection induced 


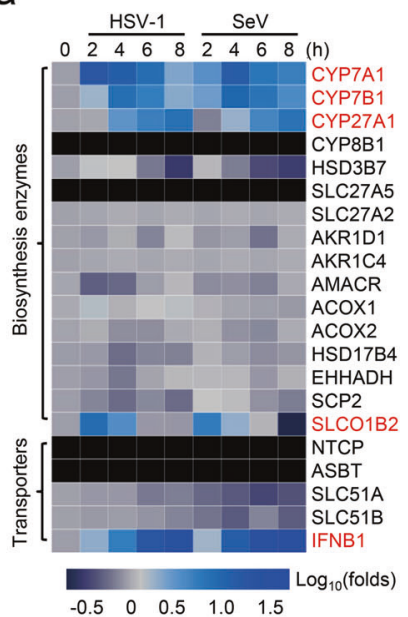

d
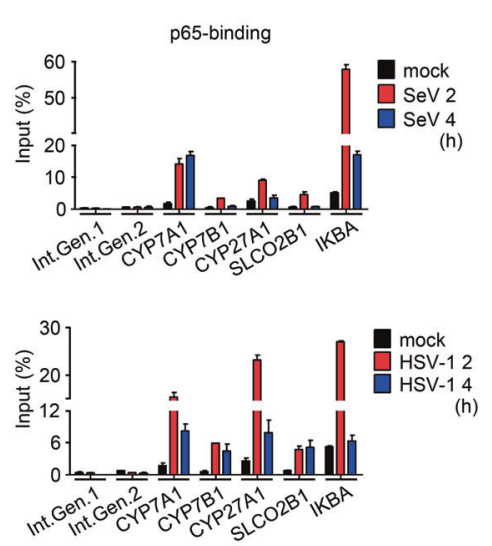

b

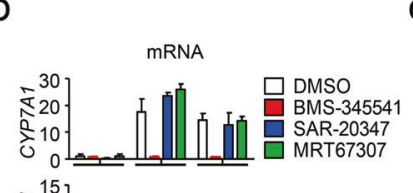

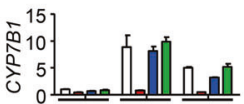

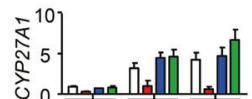

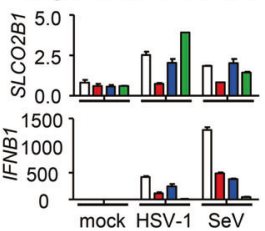

e

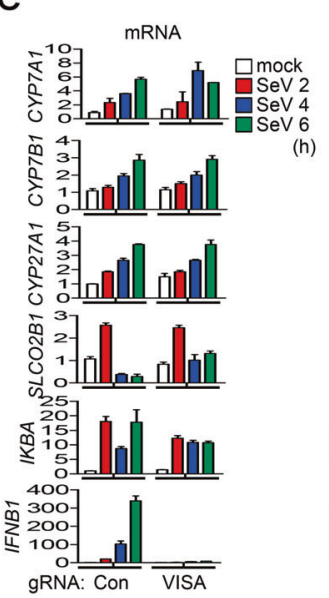

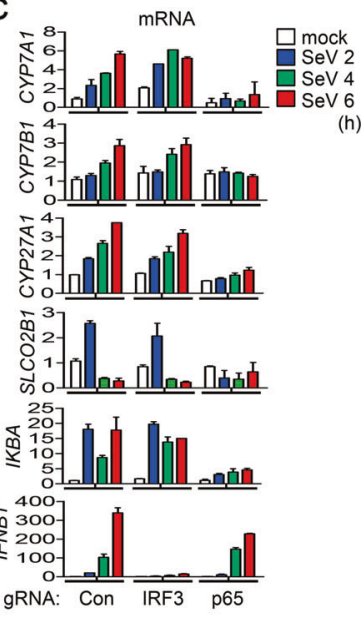

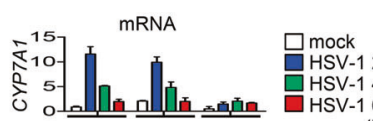
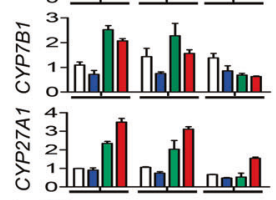

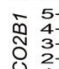

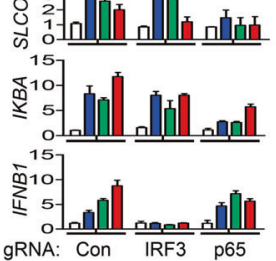

f
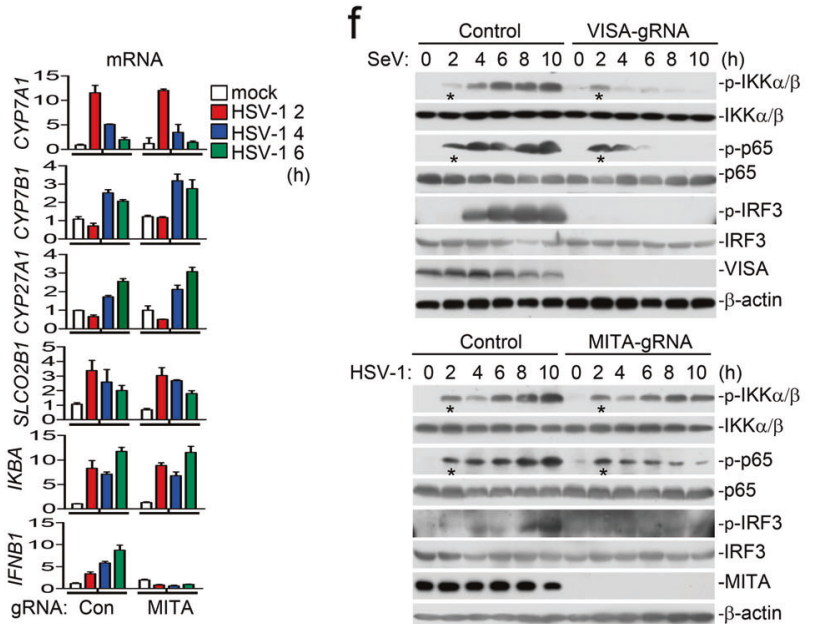

Fig. 1 Viral infection reprograms intracellular BA metabolism. a Heatmap of virus-induced transcription of genes involved in BA metabolism. THP1 cells were infected with HSV-1 or SeV for the indicated times, followed by qPCR analysis of mRNA levels of the indicated genes. The data were subjected to analysis by Heatmap. $\mathbf{b}$ Effects of kinase inhibitors on virus-induced transcription of BA transporter and rate-limiting enzyme genes. THP1 cells were treated with the indicated inhibitors followed by infection with HSV-1 or SeV for $4 \mathrm{~h}$ before qPCR analysis of the indicated mRNA levels. c Effects of IRF3 or p65 deficiency on virus-induced transcription of BA transporter and rate-limiting enzyme genes. THP1 cells stably transduced with control, gRNA of IRF3 or p65 were infected with SeV or HSV-1 as indicated for the indicated times before qPCR analysis of the indicated mRNA levels. d CHIP analysis of p65 binding to the promoters of the indicated genes. THP1 cells were infected with SeV or HSV-1 for the indicated times before CHIP assays were performed, followed by qPCR analysis of the abundance of p65-bounded DNA fragments with the indicated gene primers. e Effects of VISA or MITA deficiency on virus-induced transcription of BA transporter and ratelimiting enzyme genes. THP1 cells stably transduced with control or gRNA of VISA or MITA were infected with SeV or HSV-1 respectively as indicated for the indicated times before qPCR analysis of the mRNA levels. $f$ Effects of VISA or MITA deficiency virus-induced phosphorylation of IKK $\alpha / \beta$, p65 and IRF3. THP1 cells stably transduced with control or gRNA of VISA or MITA were infected with SeV or HSV-1 respectively for the indicated times, and cell lysates were then analyzed by immunoblots with the indicated antibodies. $n=3, \pm$ SD. See also Supplementary information, Fig. S1

biosynthesis of the primary cholic acid (CA) only in primary mouse hepatocytes but not THP-1 (Fig. 2b) or HEK293 (Supplementary information, Fig. S2d) cells. This is consistent with our results that the transcriptional level of CYP8B1, an essential enzyme for CA but not CDCA biosynthesis, was low and not induced by viral infection in THP1 cells (Fig. 1a). Next we investigated the contribution of the BA transporter and biosynthesis enzymes to virus-induced intracellular accumulation of BAs. We found that SeV- and HSV1 -induced increase of BA levels in THP1 cells cultured in CM was significantly inhibited by shRNA-mediated knockdown of the BA biosynthesis enzyme HSD3B7 (a common downstream enzyme of CYP7A1 and CYP7B1-CYP27A1 axes), or the BA transporter SLCO1B2 (Fig. 2C). When THP1 cells were cultured in BM, SeV- or HSV-1-induced increase of intracellular BA levels was significantly inhibited by knockdown of HSD3B7 but not SLCO1B2 (Fig. 2C).
However, VISA or MITA deficiency had no marked effects on virusinduced accumulation of intracellular BAs (Supplementary information, Fig. S2e), which is consistent with the previous findings that virus-induced transcription of BA transporter and biosynthesis enzyme genes is independent of VISA and MITA (Fig. 1e). These results suggest that viral infection induces accumulation of intracellular BAs by de novo biosynthesis and SLCO1B2mediated uptake from the medium in divergent types of cells.

BAs potentiate virus-induced innate immune response Since viral infection induces early rapid accumulation of intracellular BAs, we investigated its significance in innate antiviral immunity. We found that knockdown of SLCO1B2, CYP7A1, CYP7B1, CYP27A1 or HSD3B7 markedly inhibited HSV-1-1 or SeV-induced accumulation of intracellular BAs, as well as 
a
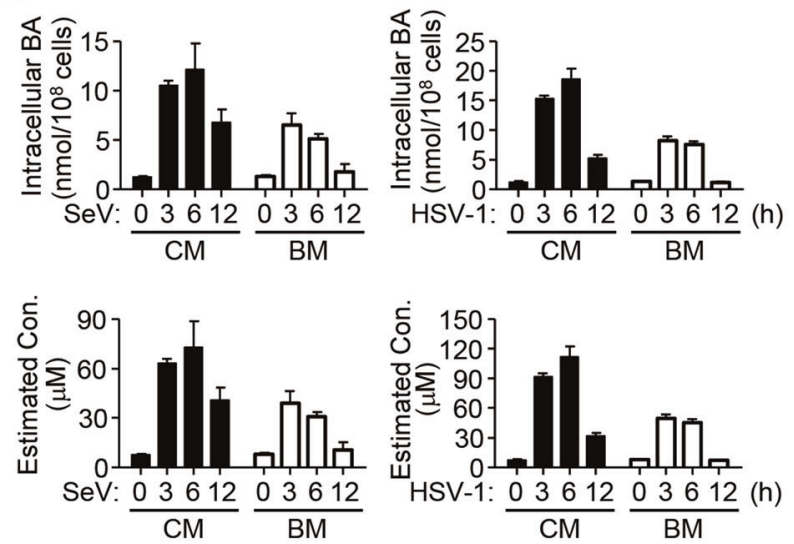

b

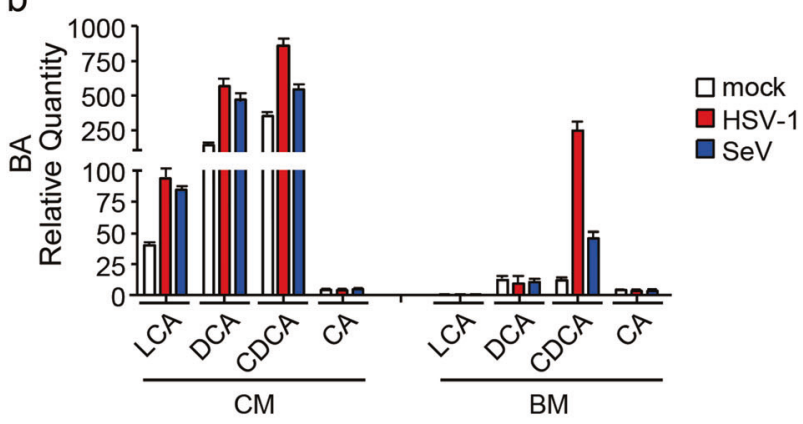

C
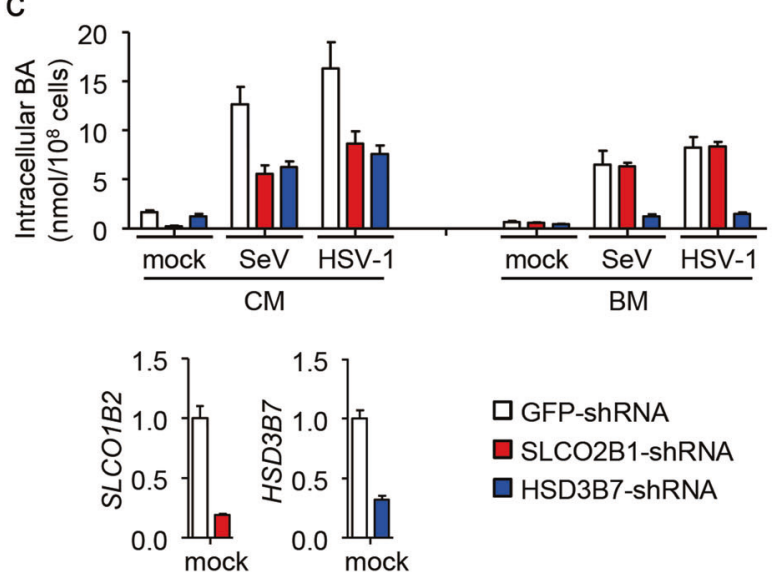

Fig. 2 Viral infection induces accumulation of intracellular BAs via both biosynthesis and uptake. a Measurement of intracellular BAs in THP-1 cells. THP1 cells $\left(1 \times 10^{8}\right)$ cultured in CM or BM were infected with $\mathrm{SeV}$ for the indicated times before measurement of intracellular BAs (upper panel), and then the estimated intracellular BA concentrations were calculated based on the diameter of a cell as $15 \mu \mathrm{m}$ (lower panel). b Analysis of intracellular BAs in THP1 cells by mass spectrometry (MS). THP1 cells cultured in CM or BM for $24 \mathrm{~h}$ were infected with SeV or HSV-1 for $6 \mathrm{~h}$ before cells were harvested for analysis of intracellular BAs by MS. c Effects of knockdown of BA transporter and rate-limiting enzymes on virus-induced accumulation of intracellular BAs. THP1 cells stably transduced with control, or shRNA for SLCO2B1or HSD3B7 were infected with HSV-1 or SeV for $6 \mathrm{~h}$ before measurement of intracellular BAs. $n=3, \pm$ SD. See also Supplementary information, Fig. S2

transcription of downstream antiviral genes IFNB1 and CXCL10 (Fig. 3a), but did not affect IFN- $\gamma$-induced transcription of IRF1 gene in THP1 cells (Supplementary information, Fig. S3a). Reporter assays indicated that knockdown of all the examined BA biosynthesis enzymes markedly inhibited transfected synthetic poly $(\mathrm{l}: \mathrm{C})$ - and B-DNA-induced activation of the IFN- $\beta$ promoter (Supplementary information, Fig. S3b and c), but had no marked effects on IFN $\gamma$-induced activation of the IRF1 promoter (Supplementary information, Fig. S3c).

We next determined whether BAs directly regulate innate antiviral response. We found that exogenous treatment of cells with LCA, CDCA and DCA, which were increased in virus-infected cells, potentiated virus-induced transcription of downstream antiviral genes including IFNB1, CXCL10 and ISG56 in mouse macrophagic Raw246.7 cells (Fig. 3b). Consistently, we detected dramatic increase of intracellular BA levels after exogenous treatment of cells with LCA, CDCA and DCA both before and after viral infection (Fig. 3c). Furthermore, CDCA treatment also potentiated SeV- or HSV-1-induced phosphorylation of IRF3 (Fig. 3d), and inhibited VSV replication (Fig. 3e) in these cells. These results suggest that BAs potentiate virus-induced innate immune response.

BAs promote innate antiviral response via the TGR5-GRK- $\beta$ arrestin-SRC axis

We next investigated the mechanisms of BA-induced innate immune response. Previous studies have shown that two BA receptors, the plasma and organelle membrane-associated TGR5 and the nuclear-located FXR, mediate multiple cellular processes in response to BAs. ${ }^{21,24}$ We found that knockdown of TGR5 but not FXR dramatically inhibited SeV-induced activation of the IFN- $\beta$ promoter, while knockdown of either TGR5 or FXR had no marked effects on IFN- $\gamma$-induced activation of the IRF1 promoter in reporter assays (Supplementary information, Fig. S4a), suggesting that TGR5 but not FXR plays an important role in BA-induced innate antiviral response. Consistently, TGR5-deficient mouse macrophages had significantly decreased levels of Ifnb1 and CxCl10 mRNAs in response to infection with HSV-1, SeV or EMCV (RNA virus), transfection with synthetic viral DNA mimics HSV120 (120-mer dsDNA representing the genome of HSV-1) or ISD45 (IFN-stimulating DNA 45), poly(I:C), or CGAMP (Fig. 4a and Supplementary information, Fig. S4b). In addition, HSV-1- and SeV-induced phosphorylation of TBK1 and IRF3 was also inhibited in TGR5-deficient macrophages (Fig. 4b). Furthermore, CDCA and INT-777, a selective TGR5 agonist, but not FXR agonist GW4064, potentiated HSV-1- and SeV-induced transcription of Ifnb1 in $\mathrm{Tgr5}^{+/+}$but not $\mathrm{Tgr}^{-/-}$macrophages (Fig. 4c). These results suggest that TGR5 plays an important role in innate antiviral response.

TGR5 is a member of G-protein coupled receptor (GPCR) family, which activates distinct downstream effector proteins including $G$ proteins and $\beta$-arrestins. We found that simultaneous but not individual knockdown of $\beta$-arrestin 1 and $\beta$-arrestin2 markedly inhibited SeV-induced activation of the IFN- $\beta$ promoter in reporter assays (Supplementary information, Fig. S4c). In the same experiments, knockdown of various subtypes of $G$ proteins including several Gai (inhibitory $G$ protein subunit a encoded by GNAI1, GNAI2 and GNAI3) and Gas (stimulatory G protein subunit a encoded by GNAS) had no effects on SeV-induced activation of the IFN- $\beta$ promoter (Supplementary information, Fig. S4c). Consistently, simultaneous knockout of $A R R B 1$ (encoding for $\beta$-arrestin1) and ARRB2 (encoding for $\beta$-arrestin2) genes by CRISPR/Cas9 dramatically inhibited HSV-1- or SeV-induced transcription of IFNB1 and CXCL10 genes in THP1 cells (Fig. $4 \mathrm{~d}$ ). These results suggest that $\beta$-arrestin1 and $\beta$-arrestin2 play redundant roles in innate antiviral response. It has been well established that GPCRrelated kinases (GRKs) are required for recruitment of $\beta$-arrestins to activated GPCR. ${ }^{26}$ We found that knockdown of GRK2, GRK4, or GRK6 markedly inhibited SeV-induced activation of the IFN- $\beta$ promoter, while knockdown of GRK3 or GRK5 had minimal effects (Supplementary information, Fig. S4d). As controls, 
a
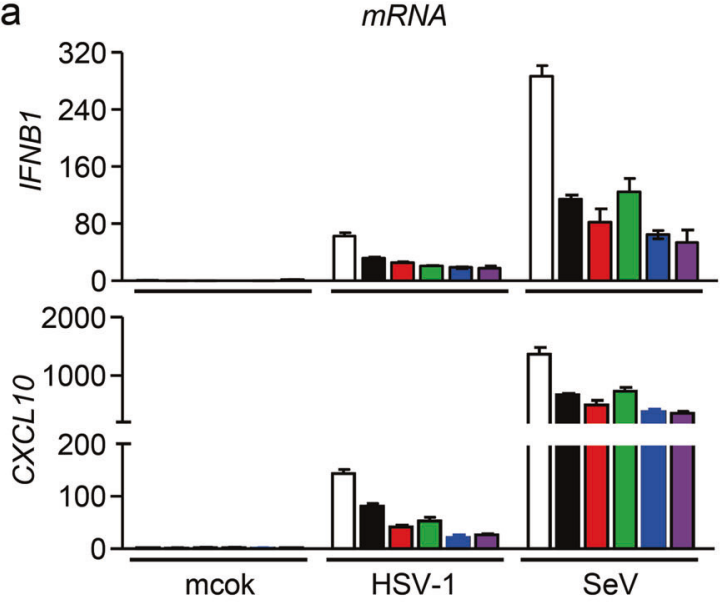

b

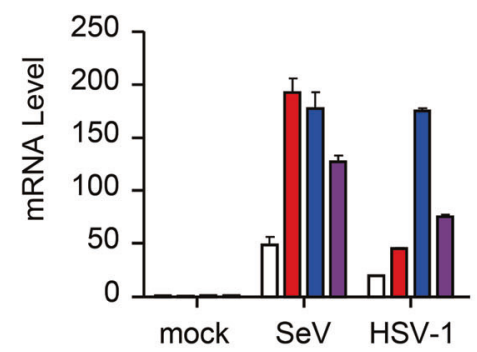

C

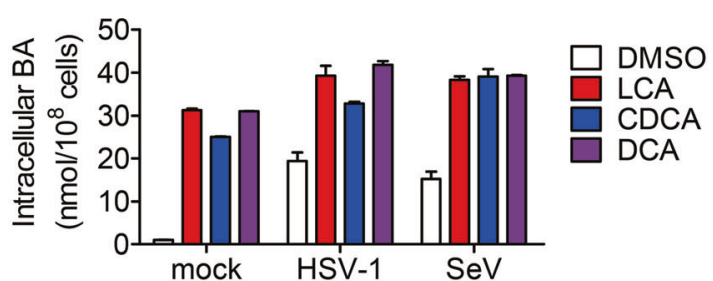

e

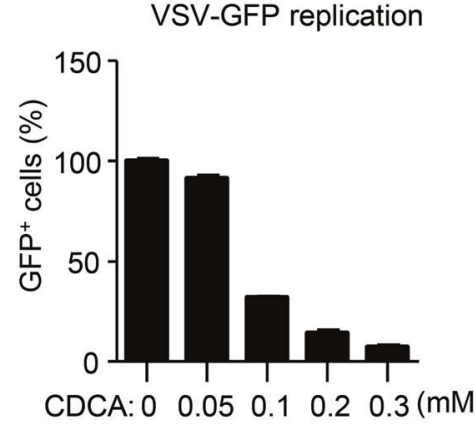

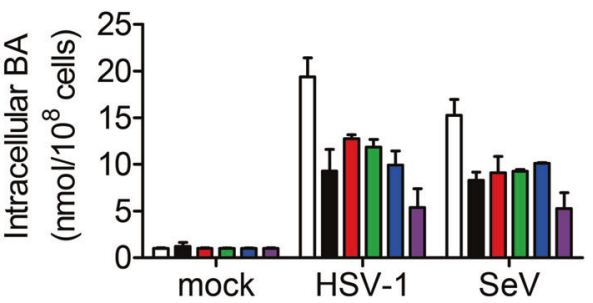

GFP-shRNA

SLCO1B2-shRNA

CYP7A1-shRNA

CYP7B1-shRNA

CYP27A1-shRNA

HSD3B7-shRNA

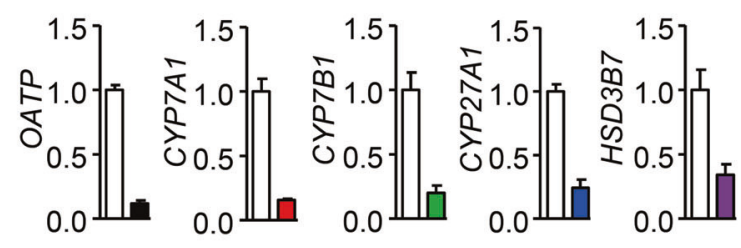

CXCL10

ISG56
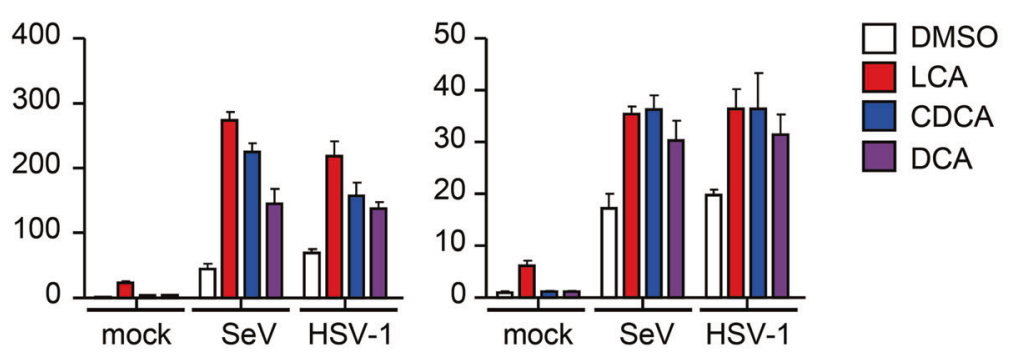

d
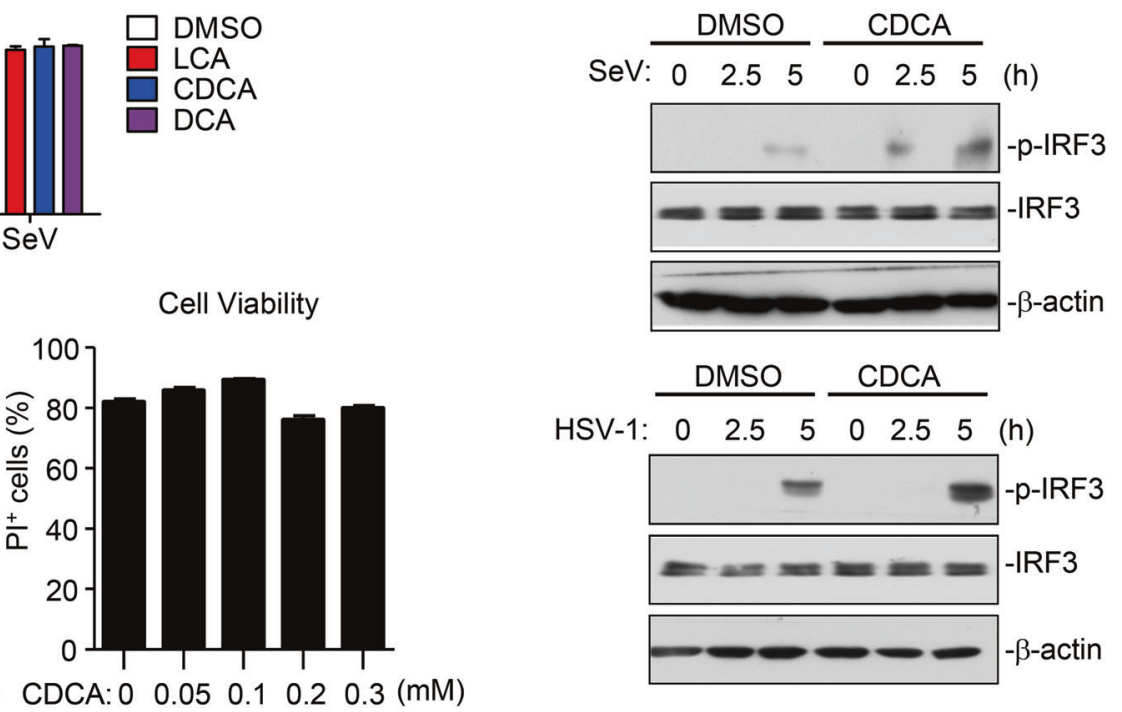

Fig. 3 Virus-triggered accumulation of intracellular BAs promotes cellular antiviral response. a Effects of SLCO2B1, CYP7A1, CYP7B1, CYP27A1 or HSD3B7 knockdown on virus-induced transcription of IFNB1 and CXCL10 genes. THP1 cells stably transduced with the indicated shRNA were infected with HSV-1 or SeV for $8 \mathrm{~h}$ before qPCR analysis of the indicated mRNAs and BA assays for intracellular BA measurements. $\mathbf{b}$ Effects of BAs on virus-induced transcription of antiviral genes. Raw264.7 cells were infected with HSV-1 or SeV for 30 min and treated with LCA, CDCA or DCA $(0.1 \mathrm{mM})$ for $6 \mathrm{~h}$ before qPCR analysis of the indicated mRNA levels. c Examination of intracellular BA levels after cells were treated with exogenous BAs. Raw264.7 cells were infected with HSV-1 or SeV for 30 min and treated with LCA, CDCA or DCA (0.1 mM) for $6 \mathrm{~h}$ before BA assays were performed. d Effects of BAs on virus-induced IRF3 phosphorylation. Raw264.7 cells were infected with HSV-1 or SeV for 30 min, treated with CDCA $(0.1 \mathrm{mM})$ for the indicated times before immunoblotting analysis with the indicated antibodies. e Effects of CDCA on VSV replication and cell viability. Raw264.7 cells were infected with VSV-GFP for $1 \mathrm{~h}$, then treated with the indicated concentrations of CDCA for 24 $\mathrm{h}$ before flow cytometry analysis of GFP-positive (GFP ${ }^{+)}$and PI-positive ( $\left.\mathrm{PI}^{+}\right)$cells. $n=3, \pm \mathrm{SD}$. See also Supplementary information, Fig. S3

knockdown of $\beta$-arrestins or GRKs had no marked effects on IFN$\gamma$-induced activation of the IRF1 promoter in reporter assays (Supplementary information, Fig. S4e). Further biochemical experiments indicated that SeV-induced phosphorylation of
TBK1 and IRF3 was markedly inhibited in $\beta$-arrestin1/2-, GRK2and GRK6-deficient cells (Supplementary information, Fig. S4f). These results suggest that the TGR5-GRK- $\beta$-arrestin axis mediates BA-triggered innate antiviral immune response. 
a

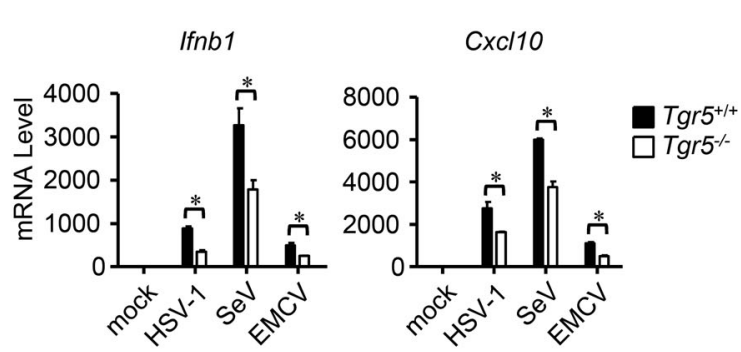

C

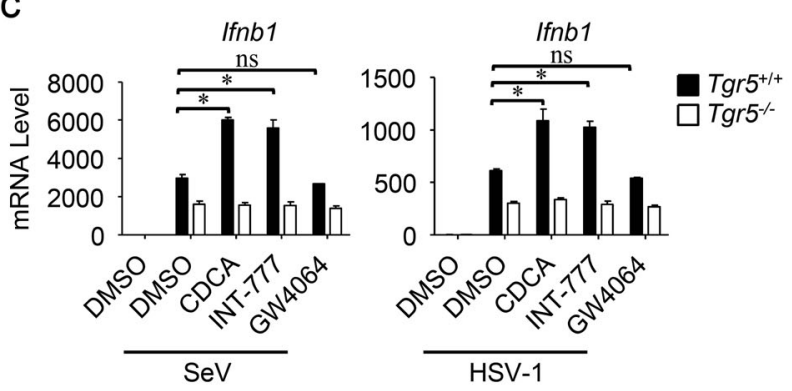

b

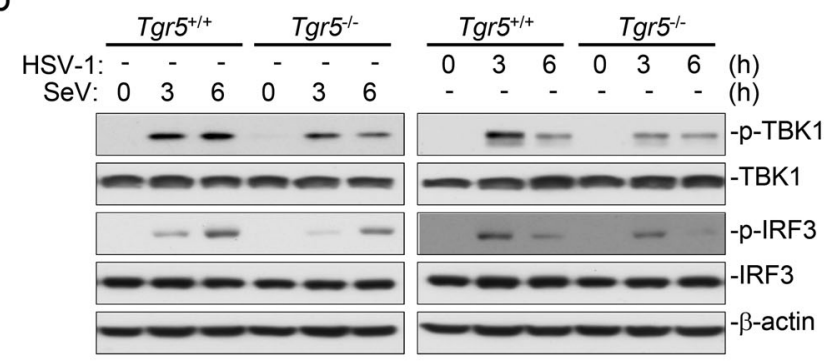

d

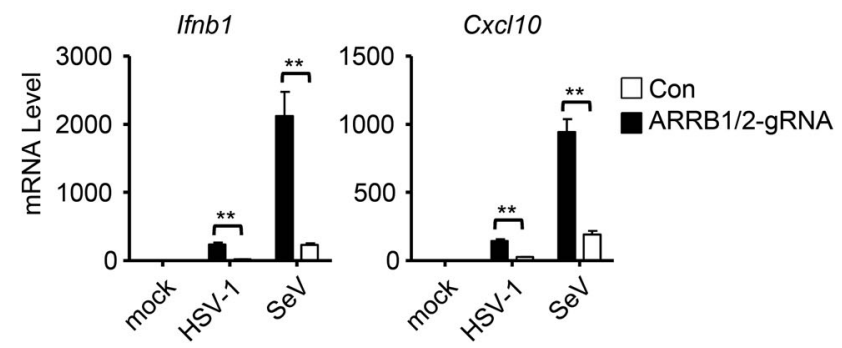

e

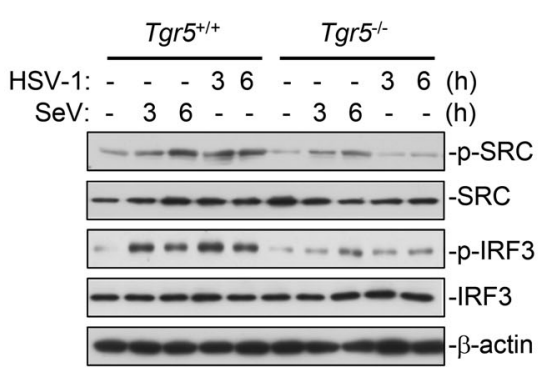

g

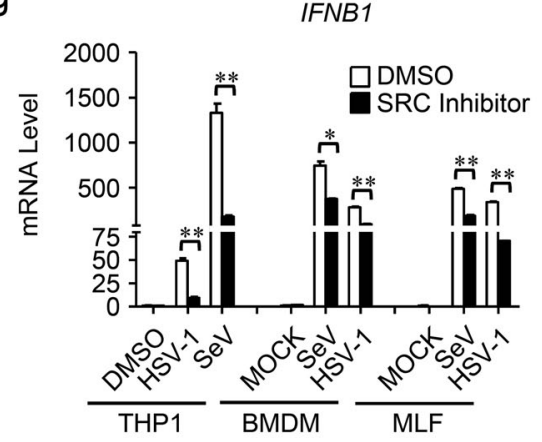

f

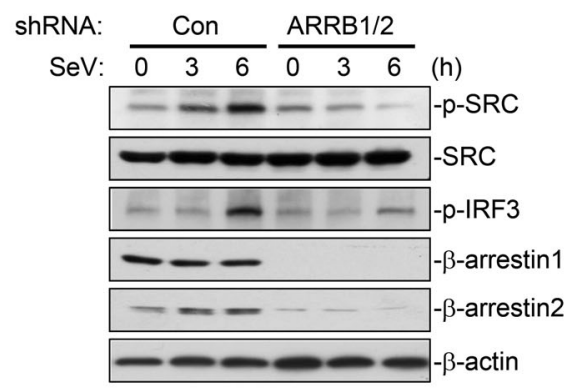

h

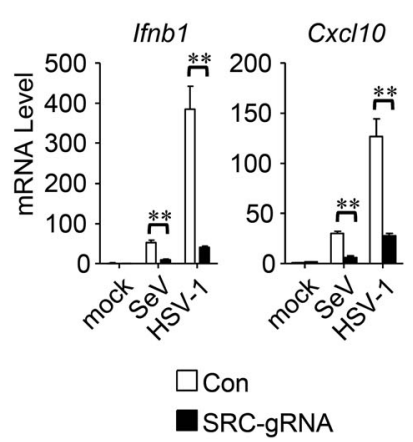

i

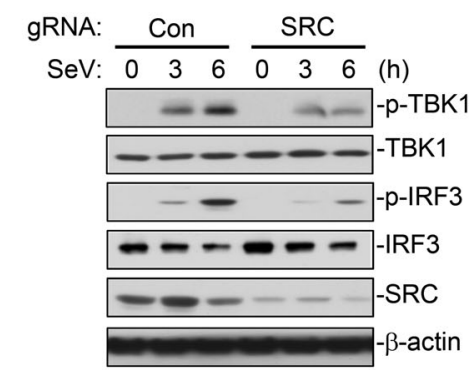

Fig. 4 BAs promote innate antiviral response via a TGR5-GRK- $\beta$-arrestin-SRC axis. a Effects of TGR5 deficiency on virus-induced transcription of Ifnb1 and $\mathrm{CxCl10.} \mathrm{Tgr}^{+/+}$and $\mathrm{Tgr}^{-/-}$BMDMs were infected with the indicated viruses for $6 \mathrm{~h}$ before qPCR analysis of the indicated mRNA levels. b Effects of TGR5 deficiency on virus-induced phosphorylation of TBK1 and IRF3. Tgr5 ${ }^{+/+}$and Tgr $5^{-1}$ BMDMs were infected with HSV-1 or SeV for the indicated times before immunoblotting analysis with the indicated antibodies. c Effects of CDCA and INT-777 on virus-induced transcription of Ifnb1. Tgr5 $5^{+/+}$and Tgr5 $^{-/-}$BMDMs were infected with HSV-1 or SeV for 30 min, then treated with CDCA, INT-777 or GW4064 for $6 \mathrm{~h}$ before qPCR analysis of Ifnb1 mRNA levels. $n=3, \pm \mathrm{SD}$. ${ }^{*} p<0.05 ;{ }^{* *} p<0.01$. d Effects of $\beta$-arrestin deficiency on virus-induced transcription of IFNB1 and CXCL10. THP1 cells stably transduced with control or gRNA of ARRB1/2 were infected with SeV or HSV-1 for $8 \mathrm{~h}$ before qPCR analysis of the indicated mRNA levels. e Effect of TGR5 deficiency on virus-induced SRC Y416 phosphorylation. Tgr5 $5^{+/+}$and Tgr5 ${ }^{-/}$BMDMs $^{-12}$ were infected with HSV-1 or SeV for the indicated times before immunoblotting analysis with the indicated antibodies. $f$ Effects of $\beta$-arrestin deficiency on virus-induced SRC Y416 phosphorylation. HEK293 cells were transfected with the indicated plasmids for $36 \mathrm{~h}$, then infected with $\mathrm{SeV}$ infection for the indicated times before immunoblotting analysis with the indicated antibodies. $\mathbf{g}$ Effects of SRC inhibitor on virus-induced transcription of IFNB1 in different cells. The indicated cells were treated with DMSO or SRC inhibitor (Dasatinib, $2 \mu M$ ), followed by viral infection for $6 \mathrm{~h}$ before qPCR analysis of IFNB1 mRNA level. h.Effects of SRC deficiency on virus-induced transcription of IFNB1 and CXCL10. MLFs stably transduced with control or SRC gRNA were infected with SeV or HSV-1 for $8 \mathrm{~h}$ before qPCR analysis of the indicated mRNA levels. i Effects of SRC deficiency on virus-induced phosphorylation of TBK1 and IRF3. MLFs stably transduced with control or SRC gRNA were infected with SeV for the indicated times before immunoblotting analysis with the indicated antibodies. $n=3, \pm$ SD. ${ }^{*} p<0.05$; ${ }^{* *} p<0.01$. See also Supplementary information, Fig. S4 
a

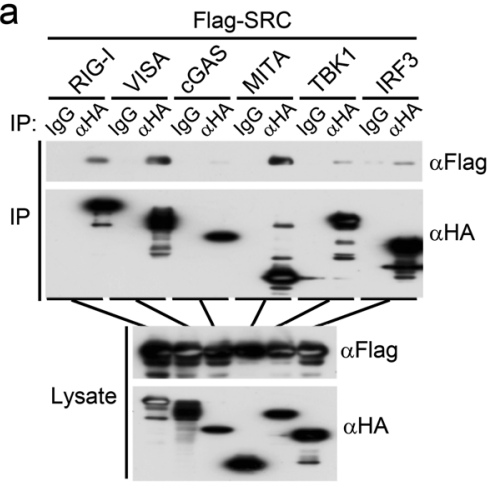

b

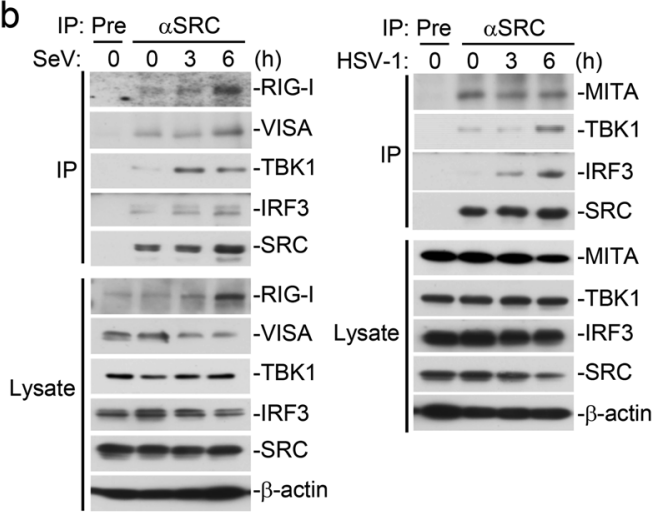

d

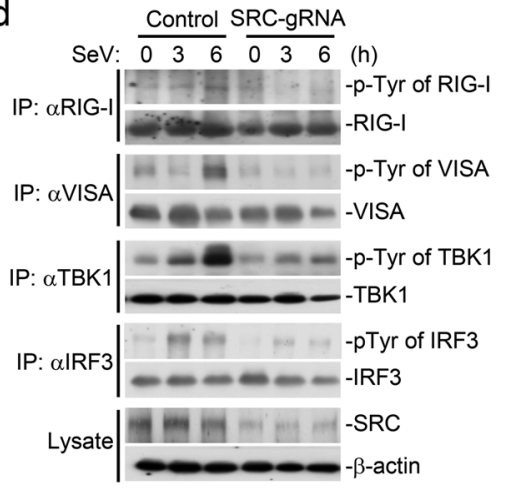

e
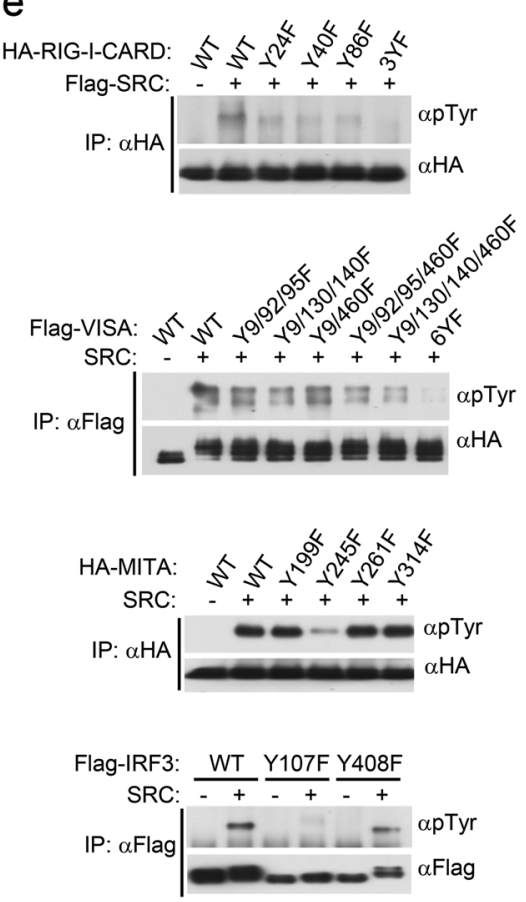

It has been reported that GRK2- and GRK6-mediated phosphorylation of $\beta$-arrestins facilitates recruitment of the non-receptor tyrosine kinase SRC to $\beta$-arrestins. ${ }^{27}$ We next determined whether SRC is activated via TGR5-GRK- $\beta$-arrestin axis in response to viral infection. Biochemical experiments indicated that HSV-1- or SeV

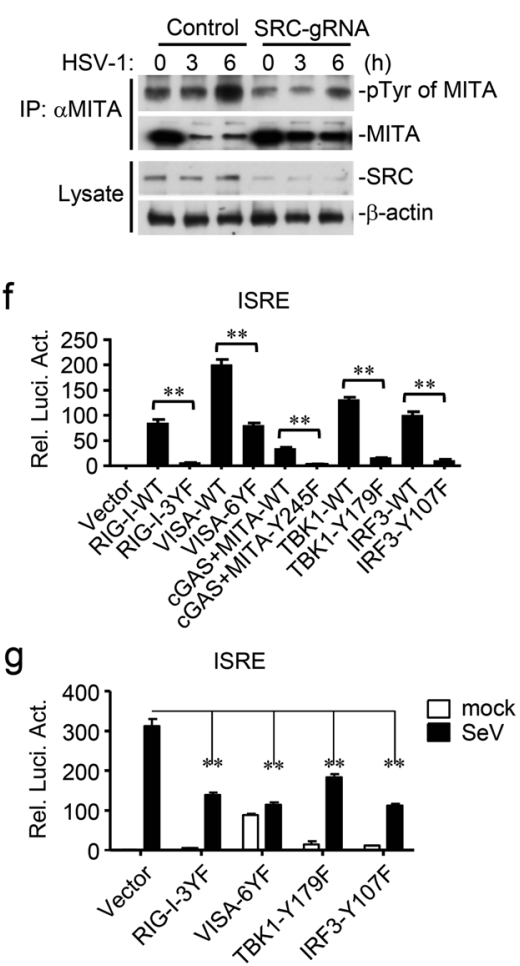

infection induced phosphorylation of SRC at Y416 in macrophages (Fig. 4e), which is a hallmark for SRC activation. ${ }^{28} \operatorname{Tgr} 5$ or $\beta$-arrestin $1 / 2$ deficiency impaired SeV-induced phosphorylation of SRC and IRF3 (Fig. 4e and f). Endogenous coimmunoprecipitation experiments indicated that $\mathrm{SeV}$ infection induced the 
Fig. 5 SRC mediates tyrosine phosphorylation of multiple innate antiviral signaling components. a Interaction of SRC with components in innate antiviral signaling pathways. HEK293 cells were transfected with the indicated plasmids for $24 \mathrm{~h}$ before coimmunoprecipitation and immunoblotting analysis with the indicated antibodies. b Endogenous association of SRC with components in innate antiviral signaling pathways. MLFs were infected with SeV or HSV-1 for the indicated times before endogenous coimmunoprecipitation and immunoblotting analysis with the indicated antibodies. c Effects of SRC on tyrosine phosphorylation of components in innate antiviral signaling pathways. HEK293 cells were transfected with the indicated plasmids for $24 \mathrm{~h}$ before coimmunoprecipitation and immunoblotting analysis with the indicated antibodies. d Effects of SRC deficiency on virus-induced tyrosine phosphorylation of components in innate antiviral signaling pathways. MLFs stably transduced with control or SRC gRNA were infected with SeV or HSV-1 for the indicated times before coimmunoprecipitation and immunoblotting analysis with the indicated antibodies. e Identification of SRC-targeted tyrosine residues of the substrates. HEK293 cells were transfected with the indicated wild-type or mutant plasmids for $24 \mathrm{~h}$ before coimmunoprecipitation and immunoblotting analysis with the indicated antibodies. $\mathbf{f}$ Effects of the indicated wild-type and mutant proteins on ISRE activation. HEK293 cells were transfected with the indicated plasmids for $24 \mathrm{~h}$ before luciferase assays. $\mathbf{g}$ Effects of the indicated mutant proteins on SeV-induced activation of ISRE. HEK293 cells were transfected with the indicated plasmids for $24 \mathrm{~h}$ and then infected with SeV for $12 \mathrm{~h}$ before luciferase assays. $n=3, \pm$ SD. ${ }^{*} p<0.05 ;{ }^{* *} p<0.01$. See also Supplementary information, Fig. S5

recruitment of $\beta$-arrestins, GRK6 and SRC to TGR5 at $3 \mathrm{~h}$ after viral infection (Supplementary information, Fig. S4g). Further experiments indicated that inhibitor of SRC but not PKA, another protein kinase activated by GPCR, suppressed virus-induced transcription of IFNB1 in THP1, mouse macrophages and fibroblasts (Fig. $4 \mathrm{~g}$ and Supplementary information, Fig. S4h). In addition, CRISPR/Cas9mediated knockout of SRC dramatically inhibited SeV-induced transcription of Ifnb1 and $\mathrm{CxCl}_{10}$ genes (Fig. 4h) as well as phosphorylation of TBK1 and IRF3 (Fig. 4i). Interestingly, treatment of progesterone, which is a natural ligand of the progestrone receptor (PGR) that activates SRC, rescued the decrease of virusinduced IFNB1 transcription in $\operatorname{Tgr5}^{-1-}$ cells (Supplementary information, Fig. S4i). Therefore, all these results suggest that TGR5-GRK- $\beta$-arrestin-mediated SRC activation is essential for efficient innate antiviral response.

SRC mediates tyrosine phosphorylation and activation of multiple innate antiviral signaling components

We next investigated whether SRC regulates key components in innate antiviral signaling pathways. Transient transfection and coimmunoprecipitation experiments indicated that SRC strongly interacted with RIG-I, VISA and MITA, and weakly interacted with TBK1 and IRF3, but had no interaction with CGAS (Fig. 5a). Endogenous coimmunoprecipitation experiments indicated that the association of SRC with RIG-I, VISA, TBK1 and IRF3 was increased after viral infection, while SRC was associated with MITA constitutively (Fig. 5b). In addition, overexpression of SRC caused tyrosine phosphorylation of RIG-I, VISA, MITA, TBK1 and IRF3 but not CGAS (Fig. 5C), whereas SRC deficiency impaired SeV- or HSV-1induced tyrosine phosphorylation of endogenous RIG-I, VISA, TBK1, IRF3 and MITA respectively (Fig. $5 \mathrm{~d}$ ). These results suggest that SRC mediates virus-induced tyrosine phosphorylation of multiple innate antiviral signaling components.

We next investigated the functional significance of SRCmediated tyrosine phosphorylation of the innate antiviral signaling components. In reporter assays, we found that overexpression of SRC enhanced RIG-I-, VISA-, MITA-, TBK1- and IRF3-mediated activation of ISRE (Supplementary information, Fig. S5a), indicating that SRC-mediated tyrosine phosphorylation regulates the antiviral activity of these molecules. Then multiple potential SRCtargeted tyrosine residues were identified for the CARD domain of RIG-I (which is responsible for signaling downstream activation), VISA, MITA and IRF3 by the GPS3.0 program (Supplementary information, Fig. S5b). Biochemical analysis showed that simultaneous mutation of $\mathrm{Y} 24, \mathrm{Y} 40$ and $\mathrm{Y} 86$ of RIG-I to phenylalanine (F) (RIG-I-CARD-3YF), simultaneous mutation of Y9, Y92, Y95, Y130, Y140 and Y460 of VISA to F (VISA-6YF), mutation of Y245 of MITA to $F(M I T A-Y / F)$, and mutation of $Y 107$ of IRF3 to $F($ IRF3-Y/F) impaired SRC-mediated tyrosine phosphorylation of these proteins (Fig. 5e), indicating that SRC catalyzes their phosphorylation at the respective residues. Reporter assays indicated that these mutants not only lost their abilities to activate ISRE in comparison to their wild-type counterparts (Fig. 5f), but also acted as dominant-negative mutants which inhibited SeV-induced activation of ISRE in reporter assays (Fig. $5 \mathrm{~g}$ ). Interestingly, mutation of $Y$ to $D$, which mimics phosphorylation of these proteins, also impaired the activation of ISRE mediated by these mutants (Supplementary information, Fig. S5c), suggesting that these residues are not only targeted for phosphorylation but are also important for structural properties of these proteins. Previously, it has been shown that tyrosine phosphorylation of TBK1 at Y179 by SRC is important for TBK1 activation. ${ }^{29}$ Taken together, these results suggest that SRC-mediated tyrosine phosphorylation of these key innate antiviral signaling components is important for their activation.

The BA-TGR5 pathway is important for host antiviral defense in vivo

Finally, we determined the importance of the BA-TGR5 axis in innate antiviral immunity in vivo. ELISA experiments indicated that HSV-1- and EMCV-induced production of serum IFN- $\beta$ and IFN- $\alpha$ was significantly inhibited in $\mathrm{Tgr}^{-/}$mice compared with their wild-type littermates (Fig. 6a). Consistently, much higher viral loads were detected in the brains of $\operatorname{Tgr}^{-1-}$ mice 3 days after infection with HSV-1 or EMCV (Fig. 6b). Furthermore, $T g r 5^{-1-}$ mice were significantly more susceptible to HSV-1- and EMCV-induced death (Fig. 6c). These results suggest that TGR5 is required for efficient host defense against viral infection in vivo. Furthermore, injection of CDCA potentiated HSV-1-induced production of IFN- $\beta$ in wild-type but not TGR5-deficient mice (Fig. 6d), and significantly suppressed viral replication in the brains (Fig. 6e) and increased the survival rate (Fig. 6f) of wild-type but not TGR5-deficient mice after HSV-1 infection. These results establish an important role of the BA-TGR5 pathway in host antiviral response.

\section{DISCUSSION}

Regulation of cellular metabolism and induction of innate immune response are two important outcomes following pathogen invasion. How cellular metabolism and innate immune response cross-talks following pathogen invasion is an unanswered question. In this study, we demonstrate that viral infection reprograms the intracellular BA metabolism, which conditions for efficient innate antiviral immune response.

Biosynthesis of BAs is believed to be exclusively restricted to the liver because the rate-limiting BA biosynthesis enzyme CYP7A1 is expressed at extremely low level in extrahepatic tissues. ${ }^{30}$ Though the rate-limiting enzymes CYP27A1 and CYP7B1 in an alternative (acidic) pathway of BA biosynthesis are also expressed in macrophages and other tissues in addition to the liver, it is unclear whether BAs can be biosynthesized via this pathway in extrahepatic tissues. In this study, we demonstrate that intracellular BAs are induced in several divergent types of cells after viral infection via transcriptional induction of the BA transporter 
a

IFN- $\beta$

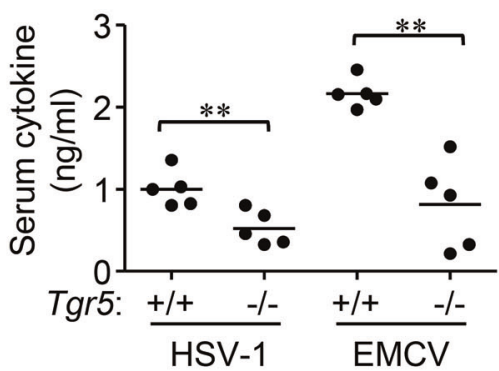

C

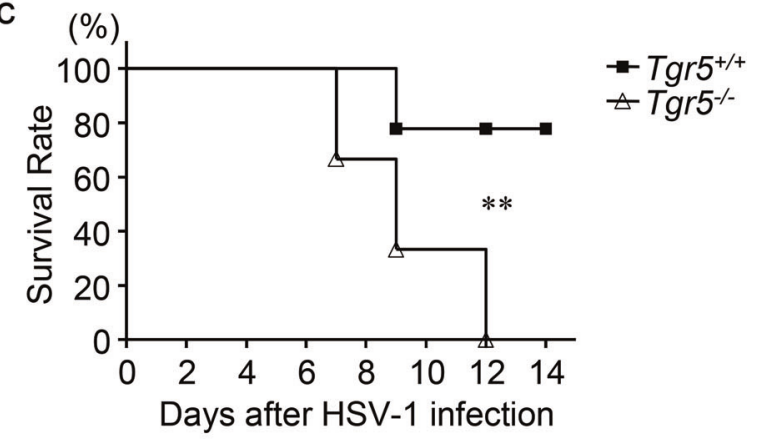

IFN- $\alpha$

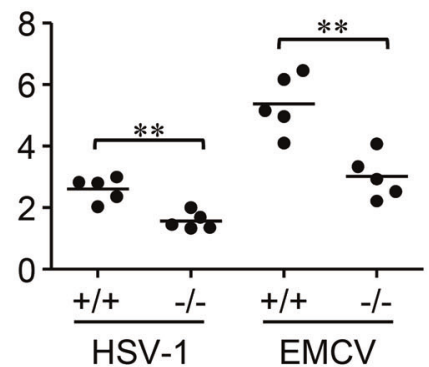

b
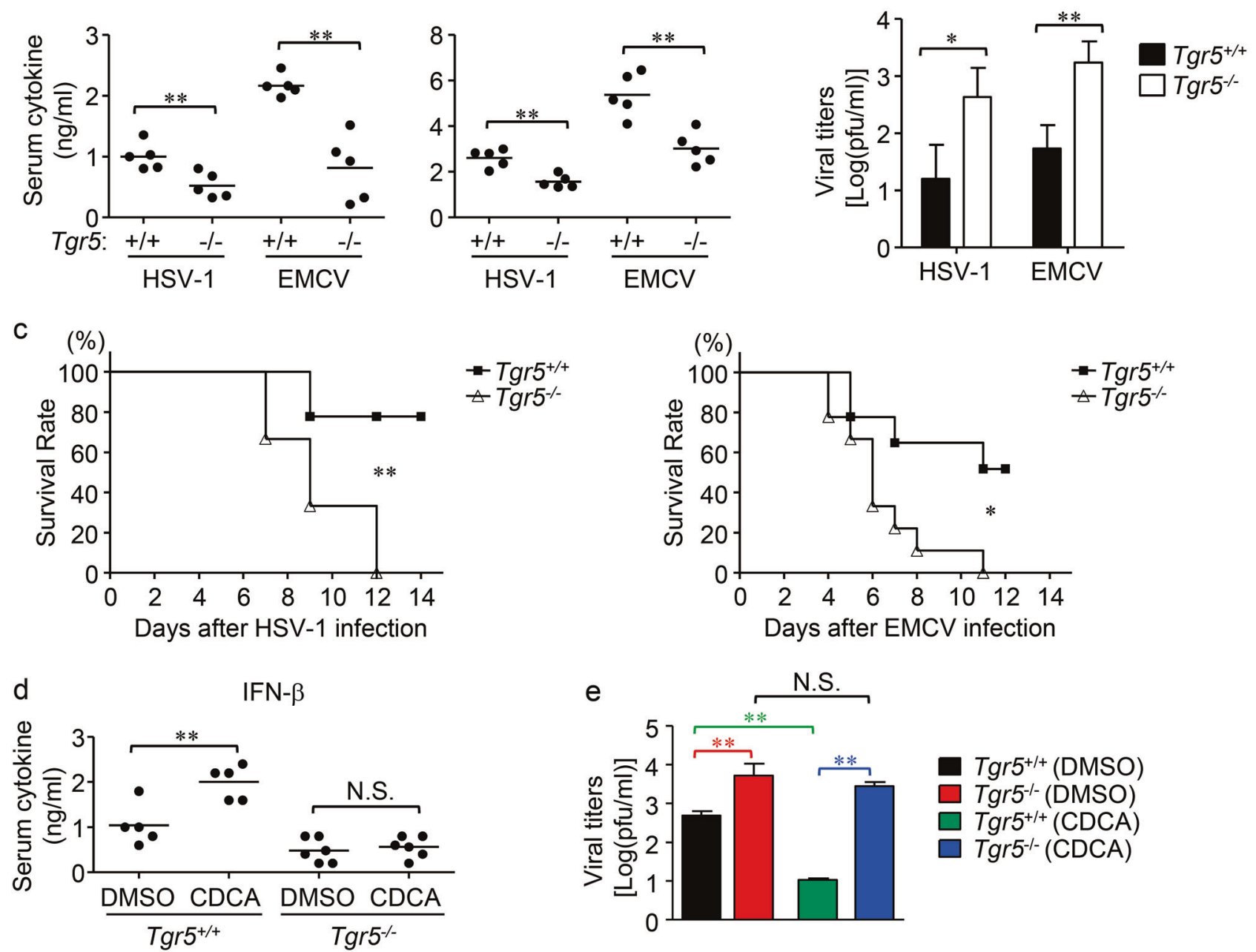

Days after EMCV infection
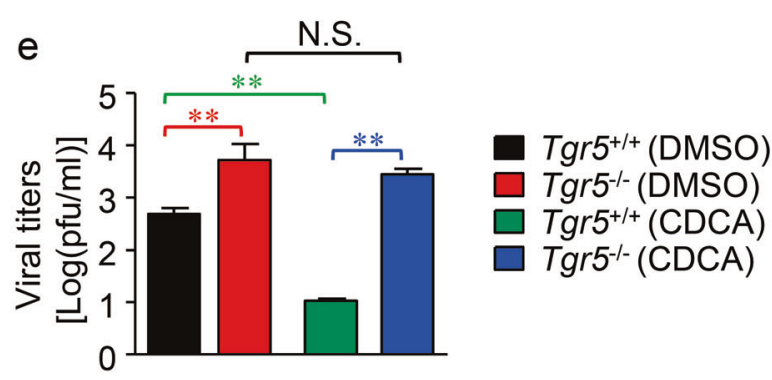

$\operatorname{Tgr} 5^{+/+}$

$\triangle \operatorname{Tgr} 5^{-1-}$

f

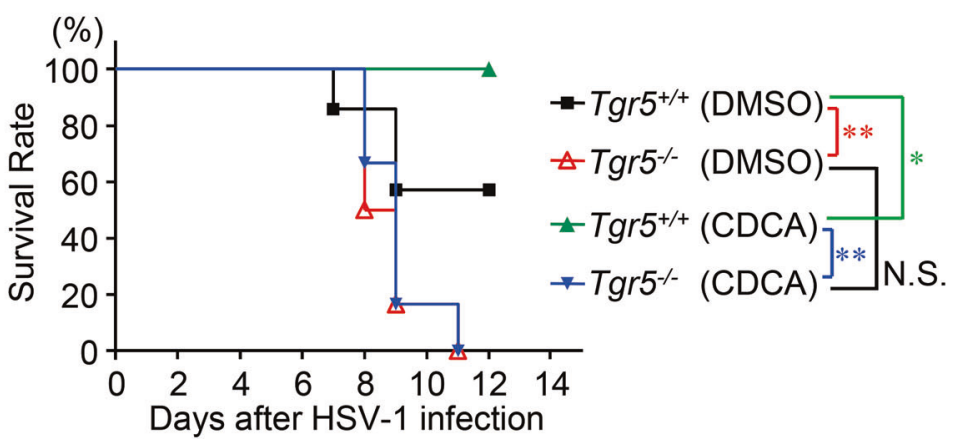

Fig. 6 The BA-TGR5 pathway is critical for host antiviral defense in vivo. a Effects of TGR5 deficiency on virus-induced production of serum cytokines. $\operatorname{Tgr}^{+/+}$and $\operatorname{Tgr} 5^{-/}$mice $(n=5)$ were infected i.p. with HSV- 1 or EMCV $\left(1 \times 10^{7}\right.$ pfu for $6 \mathrm{~h}$ before serum cytokines were measured by ELISA. b Measurements of viral titers in the brains of infected mice. $\operatorname{Tgr} 5^{+/+}$and $\operatorname{Tgr} 5^{-1-}$ mice $(n=3)$ were infected i.p. with HSV-1 $\left(1 \times 10^{7}\right.$ pfu) or EMCV $\left(1 \times 10^{6} \mathrm{pfu}\right)$, and brains were retrieved 3 days later for viral load measurement. c Survival rates of $\operatorname{Tgr5}^{+/+}$and $\operatorname{Tgr}^{-/-}$mice following viral infections. $\operatorname{Tgr}^{+/+}$and $\operatorname{Tgr}^{1 /-}$ mice $(n=12)$ were infected i.p. with HSV-1 $\left(1 \times 10^{7} \mathrm{pfu}\right)$ or EMCV $\left(1 \times 10^{6}\right.$ pfu), and the survival rates of mice were then observed and recorded for 2 weeks. d.Effects of CDCA on virus-induced production of serum cytokines. Tgr5 $5^{+/+}$and Tgr5 $5^{-1-}(n=5: 6)$ mice were infected with HSV-1 $\left(1 \times 10^{7} \mathrm{pfu}\right)$ for $1 \mathrm{~h}$ and then injected intraperitoneally with DMSO or CDCA ( $\left.30 \mathrm{mg} / \mathrm{kg}\right)$ for $6 \mathrm{~h}$ before ELISAs for serum cytokine levels. e Effects of CDCA on viral replication in the brains of mice. $\operatorname{Tgr}^{+/+}$and $\operatorname{Tgr} 5^{-1-}$ mice $(n=4)$ were infected with HSV-1 $\left(5 \times 10^{7} \mathrm{pfu}\right)$ for $1 \mathrm{~h}$ and then injected intraperitoneally with DMSO or CDCA (30 mg/kg). The mouse brains were retrieved 5 days later for viral load measurement. f.Effects of CDCA on virus-induced death of mice. $\operatorname{Tgr}^{+/+}$and $\mathrm{Tgr}^{-/-}$mice $(n=12)$ were infected with $\mathrm{HSV}-1\left(5 \times 10^{7}\right.$ pfu) for $1 \mathrm{~h}$ and then injected intraperitoneally with DMSO or CDCA (30 mg/kg). The survival rates were recorded daily for 2 weeks. $n=3, \pm S D$. ${ }^{*} p<$ $0.05 ;{ }^{* *} p<0.01 ;$ N.S. not significant 


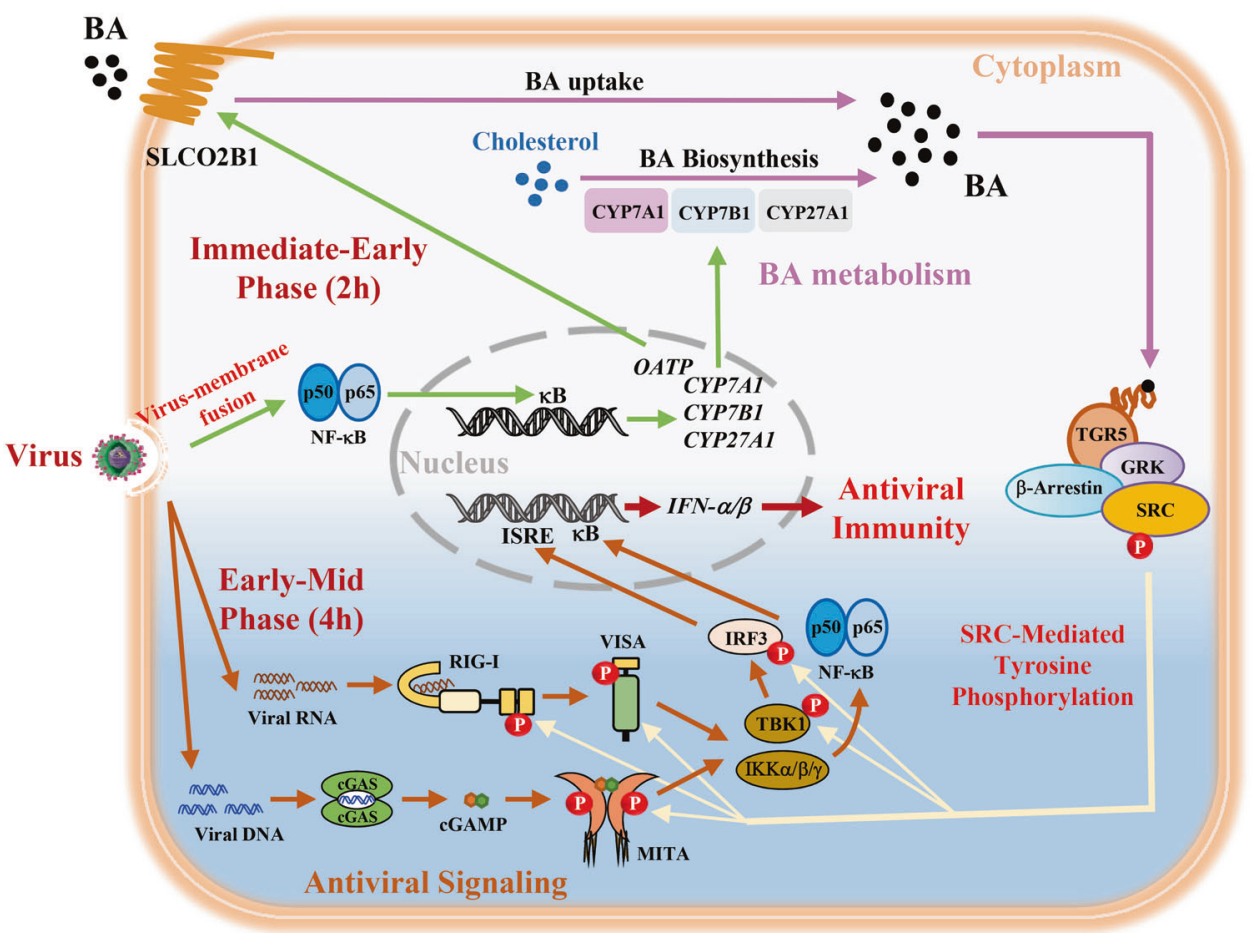

Fig. 7 A model for regulation of antiviral innate immunity by BA metabolism. Viral infection triggers an immediate-early NF- $\kappa B$ activation, which induces expression of BA transporter and rate-limiting biosynthesis enzymes, leading to accumulation of intracellular BAs by both de novo biosynthesis and uptake. The accumulated intracellular BAs activates a TGR5- $\beta$-arrestin-SRC pathway, leading to SRC-mediated tyrosine phosphorylation of several key innate antiviral signaling components, including RIG-I, VSIA, MITA, TBK1 and IRF3. The tyrosine phosphorylation of these components enables their activation by viral nucleic acid-triggered signaling, leading to innate antiviral immune response

SLCO2B1 and rate-limiting biosynthesis enzymes such as CYP7A1, CYP7B1 and CYP27A1. Since the identification of BAs several decades ago, intensive studies have revealed their hormonal functions, including direct metabolic regulation on glucose and lipid metabolism in the liver and the intestines via FXR, as well as regulation of metabolic, endocrine and neurological processes via TGR5. ${ }^{17}$ Without exceptions, all these reported functions are executed by ectocytic BAs biosynthesized and secreted from the liver or absorbed from the intestine. In this study, we found that viral infection induced accumulation of intracellular BAs via both biosynthesis and uptake, which promoted innate antiviral immune response in divergent cell types. This study reveals a universal and intrinsic cellular function of BAs in hepatic and non-hepatic cells.

Our experiments suggest that viral infection induces transcription of the BA transporter SLCO2B1 and rate-limiting biosynthesis enzyme genes, including CYP7A1, CYP7B1 and CYP27A1 in the monocytic THP1 cells. Interestingly, viral infection did not induce the examined non-rate-limiting genes in the same experiments. These results provide an explanation why a function for intracellular BAs in non-hepatic cells is not previously established. We also demonstrate that viral infection-triggered immediateearly but not VISA- or MITA-mediated NF-KB activation is responsible for transcriptional induction of the $B A$ transporter and rate-limiting biosynthesis enzyme genes. VISA or MITA deficiency inhibited SeV- or HSV-1-induced activation of NF-KB and IRF3 at $4 \mathrm{~h}$ and later phases, but had no effects on virustriggered immediate-early NF-KB activation (at $2 \mathrm{~h}$ after infection) and transcription of $B A$ genes, suggesting that virus-triggered immediate-early NF-KB activation drives the induction of the BA transporter and rate-limiting biosynthesis genes. How different viruses trigger the immediate early NF- $\mathrm{KB}$ activation is still not fully elucidated. As for HSV-1, the first rapid and transient wave of
NF-KB activation has been shown to be triggered by the binding of $\mathrm{gD}$ envelope glycoprotein to herpesvirus entry mediator $\mathrm{A}$ (HveA), a member of the TNFR superfamily, whose cytoplasmic region binds to TRAFs to activate NF-KB. ${ }^{25}$ Another enveloped virus EBV activates NF-KB via binding of its envelope glycoproteins gp350 to cellular receptor CD21 to activate downstream PI3K-AKT signaling. ${ }^{25}$ It seems that enveloped viruses perhaps activate the first-wave of NF-KB via the binding of their envelope glycoproteins to their respective receptors at the plasma membrane. As for $\mathrm{EMCV}$, a small non-enveloped virus, how it activates NF-KB at the immediate early phase is still elusive. However, a recent study suggested that sapovirus, another non-enveloped virus, activates PI3K-AKT signaling cascade via the PSaV-binding to the cell surface carbohydrate receptors. ${ }^{31}$ It is possible that EMCV activates NF-KB with similar mechanisms.

TGR5, which is a GPCR receptor for BAs, is located at both the plasma and intracellular organelle membranes. ${ }^{32}$ Our results suggest that intracellular BAs promote innate antiviral response via the TGR5- $\beta$-arrestin-SRC pathway. Firstly, deficiency of TGR5 but not FXR inhibited induction of antiviral genes triggered by BA treatment, viral infection, synthetic RNA or DNA, and cGAMP. In addition, TGR5 selective agonist INT-777 facilitates virus-induced transcription of IFNB1, while FXR agonist GW4064 had no effects on virus-induced transcription of IFNBI, indicating that BAs regulate transcription of antiviral genes through TGR5 but not FXR. Second, BAs, viral infection and TGR5 agonist INT-777 activated SRC in $\operatorname{Tgr}^{+/+}$but not $\operatorname{Tgr}^{-/-}$cells. In addition, viral infection induced the recruitment of $\beta$-arrestins and SRC to TGR5, whereas $\beta$-arrestin and SRC deficiency impaired virus-induced transcription of IFNB1 gene. Third, the intracellular levels of BAs were increased to $\sim 50-100 \mu \mathrm{M}$ following viral infection, while the extracellular levels of BAs in the medium were lower than $1.5 \mu \mathrm{M}$. 
However, exogenous addition of BAs in the culture medium could promote innate antiviral response only at high concentration $(>100 \mu \mathrm{M})$. These results suggest that TGR5 is activated by intracellular but not extracellular BAs in innate antiviral response.

Our experiments indicated that SRC mediated tyrosine phosphorylation of several key innate antiviral signaling components, including RIG-I, VISA, MITA, TBK1 and IRF3. TGR5 deficiency inhibited virus-triggered SRC activation, whereas SRC deficiency inhibited virus-triggered tyrosine phosphorylation of RIG-I, VISA, MITA, TBK1 and IRF3, activation of TBK1 and IRF3 and induction of downstream antiviral genes such as Ifnb1 and $\mathrm{CxCl}$ 10. Mutation of SRC-targeted tyrosine residues of these components to phenylalanine not only impaired their abilities to activate the IFN- $\beta$ promoter, but also made them dominant-negative mutants for virus-triggered signaling. Taken together, our results suggest that virus-triggered TGR5-dependent SRC activation and tyrosinephosphorylation of key innate antiviral signaling components condition the onset of efficient innate antiviral response. During our investigation, we noticed that basal phosphorylation of SRC at Y416 was detected in both $\operatorname{Tgr}^{+/+}$and $\operatorname{Tgr}^{-/}$cells in uninfected cells. This might account for our observations that virus-triggered induction of downstream antiviral genes was not completely lost in TGR5-deficient cells. Since SRC can be activated by distinct immune receptors, integrins and other adhesion receptors, and GPCRs, ${ }^{28,33}$ it is possible that SRC activation mediated by other pathways also contributes to innate antiviral response.

Consistent with a crucial role of the BA-TGR5 pathway in innate antiviral response, our experiments demonstrated that TGR5 deficiency inhibited HSV-1- or EMCV-induced production of serum IFN- $\beta$ and IFN- $\alpha$, increased HSV- 1 and EMCV titers in the brain, and renders the mice more susceptible to virus-induced death. Moreover, CDCA injection caused increased serum IFN- $\beta$ production in wild-type but not $\mathrm{Tgr}^{-/}$mice, and protected wild-type but not $\mathrm{Tgr}^{-/-}$mice from HSV-1-induced death. These results establish that the BA-TGR5 pathway plays an important role in host defense against viral infection in vivo.

In conclusion, our studies reveal an intrinsic role of intracellular BA-triggered metabolic pathway in innate antiviral immune response (Fig. 7). Briefly, viral infection triggers an immediateearly NF-KB activation, which results in rapid reprograming of intracellular BA metabolism including induction of BA transporter and rate-limiting biosynthesis enzymes, and increase of intracellular BAs by both de novo biosynthesis and uptake. The accumulation of intracellular BAs activates a TGR5- $\beta$-arrestin-SRC pathway, leading to SRC-mediated tyrosine phosphorylation of several key innate antiviral signaling components, including RIG-I, VSIA, MITA, TBK1 and IRF3. The tyrosine phosphorylation of these components enables their activation by viral nucleic acidtriggered signaling, leading to induction of downstream antiviral genes and innate antiviral immune response. Our findings reveal an unexpected relationship between intracellular BA metabolism and innate antiviral immune response, and point to the possibility to utilize BAs or activators of the TGR5- $\beta$-arrestin-SRC axis as antiviral agents.

\section{MATERIALS AND METHODS}

Reagents, antibodies, viruses and cells

The kinase inhibitors BMS-345541(HY-10518), SAR-20347(HY100895), MRT67307(HY-13018), INT-777(HY-15677), LCA(HYB0172), CDCA(HY-76847), DCA(HY-N0593) and CA (HY-N0324) were purchased from MedChemExpress(MCE); Dasatinib(T1448) were purchased from TargetMol; BA assay kit (MAK309) were purchased from Sigma; ISD45 and HSV120 were purchased from Sangon, poly(l:C)(t|r|-pic-5) and 2'3'-cGAMP(t|rl-nacga23-02), lipofectamine 2000 (11668019) were purchased from Invitrogen; IFN- $\gamma$ (300-02) were purchased from Peptech; ELISA kit for murine IFN- $\beta$ (42400) and IFN-a(41100) were purchased from PBL. Mouse monoclonal antibodies against HA (TA180128) were purchased from OriGene; Flag(F3165) and $\beta$-actin (A2228) antibodies were purchased from Sigma; rabbit polyclonal antibodies against SRC (2109), p-SRC(Y416)(2101), $\beta$-arrestin1(12697), $\beta$-arrestin2(3857), MITA(13647), p-p65 ${ }^{\text {S536 }}(3033), p-I K K a / \beta^{S 176 / 180}(2078)$ and IKKa/ $\beta$ and $\mathrm{p}-\mathrm{IRF}^{\mathrm{S} 396}(4947)$ were purchased from Cell signaling technology (CST); TBK1 (ab40676) and p-TBK1 ${ }^{\text {S172 }}(\mathrm{ab} 109272)$ and p65 (ab7970) were purchased from Abcam; IRF3(sc-33641) were purchased from Santa Cruz Biotechnology. SeV, EMCV, HSV-1, HCMV, VacV, NDV and VSV-GFP were previously described. ${ }^{34,35}$ HEK293 cells were obtained from ATCC. The 293T cells were originally provided by Dr. Gary Johnson (National Jewish Health). Primary $\mathrm{Tgr5}^{+/+}$and $\mathrm{Tgr5}^{-1-}$ BMDMs, and MLFs were prepared as described in previous study of MITA/STING knockout mice (Chengdu Gembio Co.,Ltd.). ${ }^{36}$

\section{Constructs}

Flag- and HA-tagged TGR5, FXR, SRC, HA-tagged RIG-I, VISA, CGAS, MITA, TBK1, IRF3 or their mutants, CRISPR-Cas9 gRNA plasmids for IRF3, p65, VISA, MITA, $\beta$-arrestin1/2 and SRC, pSuper.Retro-shRNA plasmids for CYP7A1, CYP7B1, SLCO2B1, CYP27A1, HSD3B7, AKR1D1, AMACR, ACOX1, ACOX2, HSD17B4, EHHADH, SCP2, GNAI1, GNAI2, GNAI3, GNAS, ARRB1, ARRB2, and SRC were constructed by standard molecular biology technique. The sequences targeted for gRNA and shRNA of the genes were listed in Supplementary information, Table S1.

\section{Tgr5 knockout mice}

Tgr5 knockout mice in C57/B6 background were kindly provided by Dr. Di Wang (Zhejiang University). Detailed information of Tgr5 knockout mice is previously described. ${ }^{22}$ All animal experiments were performed in accordance with the Wuhan University Medical Research Institute animal care and use committee guidelines.

\section{Cell transfection}

HEK293 and 293T cells were transfected by standard calcium phosphate precipitation method. BMDMs were transfected by lipofectamine 2000 according to procedures recommended by the manufacturer.

Measurement of intracellular BAs

For extraction of intracellular BAs, cells were washed with ice-cold PBS for three times and then mixed with $1 \mathrm{~mL}$ methanol, resuspended by vortexing for $5 \mathrm{~min}$, ultrasonicated for another $5 \mathrm{~min}$ before centrifugation at $13,680 \mathrm{~g}$ at $4^{\circ} \mathrm{C}$ for $5 \mathrm{~min}$. This procedure was repeated one more time for the pellet. For measurement of intracellular BAs by BA assay kit, the combined extracts were concentrated and then subjected to BA assay according to the instructions of the kit (Sigma, MAK309). The estimated intracellular BA concentrations were calculated based on the average diameter of the cells is $15 \mu \mathrm{m}$.

Measurement of specific types of BAs by mass spectrometry (MS) was previously described. ${ }^{37}$ Briefly, the combined supernatant was dried under nitrogen gas and reconstituted in $100 \mu \mathrm{L}$ acetonitrile followed by sequential addition of $20 \mu \mathrm{L}$ 2-chloro-1methylpyridinium iodide $(20 \mu \mathrm{mol} / \mathrm{mL}), 40 \mu \mathrm{L}$ triethylamine $(20$ $\mu \mathrm{mol} / \mathrm{mL})$ and $40 \mu \mathrm{L} \mathrm{d} 4-2$-dimethylaminoethylamine $(20 \mu \mathrm{mol} /$ $\mathrm{mL}$ ). The reaction solution was vortexed at $40^{\circ} \mathrm{C}$ for $1 \mathrm{~h}$ and then evaporated under nitrogen gas. Standard CA, LCA, CDCA, and DCA solutions labeled with 2-dimethylaminoethylamine were used as internal standards. ISs $(2 \mathrm{ng} / \mathrm{mL})$ were added to the samples followed by dissolved in $100 \mu \mathrm{L} 20 \%$ acetonitrile $(\mathrm{v} / \mathrm{v})$ and subjected for LC-ESI-MS/MS system consisting of a Shimadzu MS-8050 mass spectrometer (Tokyo, Japan) with a Shimadzu LC20AD HPLC system (Tokyo, Japan). The separation was performed on an Acquity UPLC BEH C18 column $(2.1 \times 100 \mathrm{~mm}, 1.7 \mu \mathrm{m}$, Waters) at $40^{\circ} \mathrm{C}$. The mobile phase was consisted of (A) ACN and (B) Formic acid in water $(0.1 \%, v / v)$. A gradient of $0-2$ min $80 \%$, 
2-4 $\min 80$ to $75 \% \mathrm{~B}, 4-8 \mathrm{~min} 75$ to $72 \%, 8-11 \min 72$ to $70 \%$, $11-12 \min 70$ to $40 \%$ B, $12-14 \min 40$ to $20 \%$ B, $14-15$ min 20 to $10 \% \mathrm{~B}$ and $15-16 \mathrm{~min} 10$ to $80 \% \mathrm{~B}$ was used. The flow rate of mobile phase was set as $0.4 \mathrm{~mL} / \mathrm{min}$. Multiple reaction monitoring (MRM) of $[\mathrm{M}+\mathrm{H}]+$ and the appropriate product ions were chosen to quantify BAs.

\section{qPCR}

Total RNA was isolated for qPCR analysis to measure mRNA levels of the indicated genes. Data shown are the relative abundance of the indicated mRNA normalized to that of GAPDH. qPCR primers were listed in Supplementary information, Table S1.

Coimmunoprecipitation and immunoblot analysis

Cells were lysed with RIPA buffer plus complete protease inhibitors and NEM $(20 \mathrm{mM})$, and then were sonicated for $1 \mathrm{~min}$. The lysates were centrifuged at $14,000 \mathrm{rpm}$ for $20 \mathrm{~min}$ at $4^{\circ} \mathrm{C}$. The supernatants were incubated with respective antibodies at $4{ }^{\circ} \mathrm{C}$ overnight before protein $\mathrm{G}$ beads were added for $2 \mathrm{~h}$. The beads were washed with cold PBS plus $0.5 \mathrm{M} \mathrm{NaCl}$ for three times followed by an additional wash with PBS. Proteins were separated by $8 \%$ SDS-PAGE, followed by immunoblot analysis with the indicated antibodies.

\section{Plaque assays}

To measure HSV-1 replication in mouse brains, snap-frozen brains were weighed and homogenized for 3 times (each $5 \mathrm{~s}$ ) in MEM medium. After homogenization, the brain suspensions were centrifuged at $1620 \times g$ for $30 \mathrm{~min}$, and the supernatants were used for plaque assays on monolayers of Vero cells seeded in 24well plates.

To measure HSV-1 replication in cells, cells were infected with HSV-1 $(\mathrm{MOI}=0.01)$ for the indicated times and then supernatants were collected for plaque assays on monolayers of Vero cells seeded in 24-well plates. The cells were infected by incubation for $1 \mathrm{~h}$ at $37^{\circ} \mathrm{C}$ with serial dilutions of brain suspensions. After infection for $1 \mathrm{~h}, 2 \%$ methylcellulose was overlaid, and the plates were incubated for about $48 \mathrm{~h}$. The overlay was removed and cells were fixed with $4 \%$ paraformaldehyde for $15 \mathrm{~min}$ and stained with $1 \%$ crystal violet for 30 min before plaque counting.

\section{Statistical analysis}

For mouse experiments, no specific blinding method was used, but mice in each group were selected randomly. The sample size (n) of each experimental group is described in the corresponding figure legend. GraphPad Prism software was used for all statistical analyses. Quantitative data in histograms are shown as means \pm SD. Data were analyzed using a Student's unpaired $t$-test or Logrank (Mantel-Cox) test. The number of asterisks represents the degree of significance with respect to $P$ values. Statistical significance was set at $P<0.05$.

\section{ACKNOWLEDGEMENTS}

We thank Dr. Rui Xiao for his assistant on CHIP assays analysis. This work was supported by grants from the State Key R\&D Program of China (2017YFA0505800, 2016YFA0502102), and the National Natural Science Foundation of China (31830024, 31771555, 31630045, 31521091, 31671465, and 31800728), National Postdoctoral Program for Innovative Talents (BX201600116) and China Postdoctoral Science Foundation (2017M620334).

\section{AUTHOR CONTRIBUTIONS}

H.B.S. and M.M.H. conceived and designed the study; M.M.H., W.R.H., P.G., Q.Y., K.H., and L.B.C. performed the experiments; H.B.S., M.M.H., Q.Y., S.L. and Y.Q.F. analyzed the data. M.M.H. and H.B.S. wrote the manuscript.

\section{ADDITIONAL INFORMATION}

Supplementary information accompanies this paper at https://doi.org/10.1038/ s41422-018-0136-1.

Competing interests: The authors declare no competing interests.

\section{REFERENCES}

1. Takeuchi, O. \& Akira, S. Pattern recognition receptors and inflammation. Cell 140, $805-820$ (2010)

2. Crowl, J. T., Gray, E. E., Pestal, K., Volkman, H. E. \& Stetson, D. B. Intracellular nucleic acid detection in autoimmunity. Annu. Rev. Immunol. 35, 313-336 (2017).

3. Hu, M. M. \& Shu H. B. Cytoplasmic mechanisms of recognition and defense of microbial nucleic acids. Annu. Rev. Cell Dev. Biol. 34, 357-379 (2018).

4. Seth, R. B., Sun, L., Ea, C. K. \& Chen, Z. J. Identification and characterization of MAVS, a mitochondrial antiviral signaling protein that activates NF-kappaB and IRF 3. Cell 122, 669-682 (2005).

5. Kawai, T. et al. IPS-1, an adaptor triggering RIG-I- and Mda5-mediated type I interferon induction. Nat. Immunol. 6, 981-988 (2005).

6. $\mathrm{Xu}, \mathrm{L}$. G. et al. VISA is an adapter protein required for virus-triggered IFN-beta signaling. Mol. Cell 19, 727-740 (2005).

7. Meylan, E. et al. Cardif is an adaptor protein in the RIG-I antiviral pathway and is targeted by hepatitis C virus. Nature 437, 1167-1172 (2005).

8. Sun, L., Wu, J., Du, F., Chen, X. \& Chen, Z. J. Cyclic GMP-AMP synthase is a cytosolic DNA sensor that activates the type I interferon pathway. Science 339, 786-791 (2013).

9. Gao, P. et al. Cyclic $\left[G\left(2^{\prime}, 5^{\prime}\right) p A\left(3^{\prime}, 5^{\prime}\right)\right.$ p] is the metazoan second messenger produced by DNA-activated cyclic GMP-AMP synthase. Cell 153, 1094-1107 (2013).

10. Zhong, B. et al. The adaptor protein MITA links virus-sensing receptors to IRF3 transcription factor activation. Immunity 29, 538-550 (2008).

11. Ishikawa, H., Ma, Z. \& Barber, G. N. STING regulates intracellular DNA-mediated, type I interferon-dependent innate immunity. Nature 461, 788-792 (2009).

12. Cai, X., Chiu, Y. H. \& Chen, Z. J. The cGAS-cGAMP-STING pathway of cytosolic DNA sensing and signaling. Mol. Cell 54, 289-296 (2014).

13. Chiang, C. \& Gack, M. U. Post-translational control of intracellular pathogen sensing pathways. Trends Immunol. 38, 39-52 (2017).

14. Ganeshan, K. \& Chawla, A. Metabolic regulation of immune responses. Annu. Rev. Immunol. 32, 609-634 (2014).

15. Kelly, B. \& O'Neill, L. A. Metabolic reprogramming in macrophages and dendritic cells in innate immunity. Cell Res. 25, 771-784 (2015).

16. Russell, D. W. Fifty years of advances in bile acid synthesis and metabolism. J. Lipid Res. 50, S120-S125 (2009).

17. de Aguiar Vallim, T. Q., Tarling, E. J. \& Edwards, P. A. Pleiotropic roles of bile acids in metabolism. Cell. Metab. 17, 657-669 (2013).

18. Kawamata, Y. et al. A G protein-coupled receptor responsive to bile acids. J. Biol. Chem. 278, 9435-9440 (2003).

19. Maruyama, T. et al. Identification of membrane-type receptor for bile acids (MBAR). Biochem. Biophys. Res. Commun. 298, 714-719 (2002).

20. Pols, T. W., Noriega, L. G., Nomura, M., Auwerx, J. \& Schoonjans, K. The bile acid membrane receptor TGR5 as an emerging target in metabolism and inflammation. J. Hepatol. 54, 1263-1272 (2011).

21. Gadaleta, R. M., Cariello, M., Sabba, C. \& Moschetta, A. Tissue-specific actions of FXR in metabolism and cancer. Biochim. Biophys. Acta 1851, 30-39 (2015).

22. Guo, C. et al. Bile acids control inflammation and metabolic disorder through inhibition of NLRP3 inflammasome. Immunity 45, 802-816 (2016).

23. Patman, G. Hepatitis: HBV infection alters bile acid metabolism gene profile. Nat. Rev. Gastroenterol. Hepatol. 11, 332 (2014).

24. Perino, A. \& Schoonjans, K. TGR5 and immunometabolism: insights from physiology and pharmacology. Trends Pharmacol. Sci. 36, 847-857 (2015).

25. Santoro, M. G., Rossi, A. \& Amici, C. NF-kappaB and virus infection: who controls whom. EMBO J. 22, 2552-2560 (2003).

26. Premont, R. T. \& Gainetdinov, R. R. Physiological roles of $G$ protein-coupled receptor kinases and arrestins. Annu. Rev. Physiol. 69, 511-534 (2007).

27. Yang, F. et al. Phospho-selective mechanisms of arrestin conformations and functions revealed by unnatural amino acid incorporation and (19)F-NMR. Nat. Commun. 6, 8202 (2015)

28. Cooper, J. A. \& Howell, B. The when and how of Src regulation. Cell 73, 1051-1054 (1993).

29. Li, X., et al. The tyrosine kinase Src promotes phosphorylation of the kinase TBK1 to facilitate type I interferon production after viral infection. Sci. Signal 10, eaae0435 (2017).

30. Russell, D. W. The enzymes, regulation, and genetics of bile acid synthesis. Annu. Rev. Biochem. 72, 137-174 (2003). 
31. Soliman, M., et al. Phosphatidylinositol 3-Kinase/Akt and MEK/ERK signaling pathways facilitate sapovirus trafficking and late endosomal acidification for viral uncoating in LLC-PK cells. J. Virol. 92, e01674-18 (2018).

32. Masyuk, A. I. et al. Ciliary subcellular localization of TGR5 determines the cholangiocyte functional response to bile acid signaling. Am. J. Physiol. Gastrointest. Liver Physiol. 304, G1013-G1024 (2013).

33. Schlessinger, J. New roles for Src kinases in control of cell survival and angiogenesis. Cell 100, 293-296 (2000).

34. Hu, M. M., Liao, C. Y., Yang, Q., Xie, X. Q. \& Shu, H. B. Innate immunity to RNA virus is regulated by temporal and reversible sumoylation of RIG-I and MDA5. J. Exp. Med. 214, 973-989 (2017).
35. Hu, M. M. et al. Sumoylation promotes the stability of the DNA sensor cGAS and the adaptor STING to regulate the kinetics of response to DNA virus. Immunity $\mathbf{4 5}$ 555-569 (2016).

36. Hu, M. M. et al. TRIM38 negatively regulates TLR3/4-mediated innate immune and inflammatory responses by two sequential and distinct mechanisms. J. Immunol. 195, 4415-4425 (2015).

37. Zhu, Q. F. et al. Analysis of cytochrome P450 metabolites of arachidonic acid by stable isotope probe labeling coupled with ultra high-performance liquid chromatography/mass spectrometry. J. Chromatogr. A 1410, 154-163 (2015). 